\title{
Partial Spectral Projected Gradient Method with Active-Set Strategy for Linearly Constrained Optimization *
}

\author{
Marina Andretta ${ }^{\dagger} \quad$ Ernesto G. Birgin ${ }^{\dagger} \quad$ J. M. Martínez ${ }^{\ddagger}$
}

March 9, 2009

\begin{abstract}
A method for linearly constrained optimization which modifies and generalizes recent box-constraint optimization algorithms is introduced. The new algorithm is based on a relaxed form of Spectral Projected Gradient iterations. Intercalated with these projected steps, internal iterations restricted to faces of the polytope are performed, which enhance the efficiency of the algorithms. Convergence proofs are given and numerical experiments are included and commented. Software supporting this paper is available through the TANGO Project web page: http://www.ime.usp.br/ egbirgin/tango/.
\end{abstract}

Key words: Linearly constrained optimization, spectral projected gradient method, active set methods.

\section{Introduction}

The seminal 1988 paper of Barzilai and Borwein [8] on alternative steplengths for the steepest descent method gave rise to a lot of computational optimization research.

The Spectral Projected Gradient (SPG) method was introduced, analyzed and implemented in $[17,18,19]$. This method combines the basic spectral-step ideas $[8,53,54]$ with projected gradient strategies $[10,37,45]$. The extension of the spectral gradient method to smooth convex programming problems $[5,15,14,17,18,19]$ was motivated by the surprisingly good performance of the spectral gradient for large-scale unconstrained minimization [54]. Nonmonotone strategies, like the one proposed by Grippo, Lampariello and Lucidi [40], turned out to be an important ingredient for the success of the spectral idea for unconstrained minimization and other extensions.

The SPG method is applicable to convex constrained problems in which the projection onto the feasible set is easy to compute. It has been intensively used in many different applied problems $[9,11,13,22,29,43,44,52,58,60,65]$. Several interesting parameter modifications were proposed in the papers $[24,23,26,27,33,41,55,64]$. Alternative nonmonotone strategies

\footnotetext{
*This work was supported by PRONEX-Optimization (PRONEX - CNPq / FAPERJ E-26 / 171.510/2006 APQ1), FAPESP (Grant 2006/53768-0) and CNPq.

${ }^{\dagger}$ Department of Computer Science, Institute of Mathematics and Statistics, University of São Paulo, Rua do Matão 1010 Cidade Universitária, 05508-090 São Paulo SP, Brazil. (\{andretta|egbirgin\}@ime.usp.br)

${ }^{\ddagger}$ Department of Applied Mathematics, IMECC-UNICAMP, CP 6065, 13081-970 Campinas SP, Brazil. (martinez@ime.unicamp.br)
} 
have been suggested in $[24,27,28,59,66]$, whereas convergence and stability properties were studied in $[25,32,62,63]$. In addition, SPG has been combined with other algorithms for particular optimization problems. Bound constrained minimization was addressed in $[6,14,15]$. Linearly constrained optimization and nonlinear systems were considered in [5, 48] and [42, 67], respectively. In [48] a penalty approach was used and in [5] problems were solved by means of sequential quadratic programming. For general nonlinear programming, a method combining SPG with the augmented Lagrangian was proposed in [30], and augmented Lagrangian methods that use the methods introduced in $[6,15]$ to solve bound constrained subproblems were developed in [3, 4, 12]. More recently, Gomes-Ruggiero, Martínez and Santos [38] showed that the Inexact Restoration framework $[39,46,47]$ motivates a natural generalization of SPG to general constrained optimization.

The main drawback for the application of SPG to arbitrary convex (in particular, linearly constrained) problems is the expensiveness of computing projections in general cases. In the present research, in order to apply SPG ideas to general linearly constrained optimization, we used two different strategies:

1. Enlarging the set onto which projections must be computed.

2. Combining the SPG-like iterations with easy-to-compute "essentially unconstrained" iterations which provide practical efficiency to the method.

The enlargement of the projection set involves elimination of constraints. So, by means of this procedure, projections become less expensive. Radical elimination would involve preserving only active constraints in the projection set, but this elimination would be inefficient and even convergence proofs would be impossible. A careful management of the constraints that must be preserved at each state of the calculation gives rise to the Partial Spectral Projected Gradient (PSPG) strategy introduced in the present paper.

The strategy of combining SPG-like iterations with iterations restricted to proper faces was already used in $[6,14,15]$ in connection to box-constrained optimization. The main ideas of the present research are inherited from the box-constrained ideas of $[6,15]$ but the consideration of general linear constraints, instead of merely bounds, imposes different algorithmic decisions. In the case of boxes, projections are trivially computed, therefore it is inexpensive to test, at every iteration, if the face where the current approximation lies must be preserved or not, using the comparison of internal and external components of the constrained steepest descent direction [15]. In the case of linear constraints we prefer to preserve the same active set until the boundary is reached or an approximate active-face constrained stationary point occurs. In this way we reduce, as much as possible, projection steps. The idea of combining PSPG steps with internal iterations can also be understood from the point of view of active-set methods. In Rosen's gradient projection method [56] (one of the first algorithms to which the active-set paradigm applies), and many variations reported in text books, the procedure for giving up active constraints involves removing one constraint per iteration (as in the Simplex method) and, usually, no more than one constraint is added to the working set when the current point hits the boundary. Removing many constraints per iteration is guaranteed in the algorithm presented in the current paper by the PSPG procedure. The possibility of adding constraints very slowly (possibly because of primal degeneracy) is monitored and also corrected by means of PSPG. 
This paper is organized as follows. In Section 2 we define the problem, faces and projections that will be used in the algorithms. In Section 3 we present the main algorithm, the PSPG iteration and the Internal Algorithm. The assumptions that must be fulfilled by the internal procedures and a practical scheme to define an Internal Algorithm starting from an unconstrained method are also described. Convergence of the main algorithm is proved in Section 4. In Section 5 we discuss the practical implementation of the algorithms. Numerical experiments are reported in Section 6. In the last section we state some conclusions.

\section{Notation}

We denote $\mathbb{N}=\{0,1,2, \ldots\}$.

If $v, w \in \mathbb{R}^{n}$, we denote

$$
[v, w]=\left\{u \in \mathbb{R}^{n} \mid u=t v+(1-t) w \text { for some } t \in[0,1]\right\}
$$

and

$$
v_{+}=\left(\max \left\{v_{1}, 0\right\}, \ldots, \max \left\{v_{n}, 0\right\}\right)^{T} .
$$

If $v, w \in \mathbb{R}^{n}$, the statement $v \leq w$ means that $v_{i} \leq w_{i}$ for all $i=1, \ldots, n$. We denote $P_{A}(v)$ the Euclidian projection of $v$ onto the set $A$. The symbol $\|\cdot\|$ denotes the Euclidian norm, although many times may be replaced by an arbitrary norm. The closure of the set $A$ will be denoted, as usually, by $\bar{A}$.

\section{Statement of the problem}

Let $f: \mathbb{R}^{n} \rightarrow \mathbb{R}$ be continuously differentiable. Let $\Omega \subset \mathbb{R}^{n}$ be defined by:

$$
\Omega=\left\{x \in \mathbb{R}^{n} \mid C_{i}(x) \leq 0, i=1, \ldots, p\right\},
$$

where, for all $i=1, \ldots, p$,

$$
C_{i}(x)=\left(a^{i}\right)^{T} x-b_{i}
$$

and

$$
a^{i}=\left(a_{1}^{i}, \ldots, a_{n}^{i}\right)^{T} .
$$

The problem addressed here is

$$
\text { Minimize } f(x) \text { subject to } C_{i}(x) \leq 0, i=1, \ldots, p \text {. }
$$

Given $I \subset\{1, \ldots p\}$ we denote

$$
F_{I}=\left\{x \in \Omega \mid C_{i}(x)=0 \text { if and only if } i \in I\right\} .
$$

Clearly, $\Omega$ is the union of the sets $F_{I}$, for $I \subset\{1, \ldots, p\}$. Moreover, $F_{I} \neq F_{J}$ implies that $F_{I} \cap F_{J}=\emptyset$. The smaller affine subspace that contains a nonempty face $F_{I}$ will be denoted by $V_{I}$ and the parallel linear subspace to $V_{I}$ will be $S_{I}$.

Given $z \in \Omega$ and $\delta \in[0, \infty]^{p}$, we define

$$
\Omega(z, \delta)=\left\{x \in \mathbb{R}^{n} \mid C_{i}(x) \leq 0 \text { for all } i \in I(z, \delta)\right\},
$$


where

$$
I(z, \delta)=\left\{i \mid C_{i}(z) \geq-\delta_{i}\right\} .
$$

Clearly, $\Omega \subset \Omega(z, \delta)$ and $\Omega(z, \infty)=\Omega$. Observe that, if $z \in \Omega$ satisfies the KKT conditions of (2) then it also satisfies the KKT conditions of

$$
\text { Minimize } f(x) \text { subject to } x \in \Omega(z, \delta)
$$

for all $\delta \geq 0$. Reciprocally, if $z$ satisfies the KKT conditions of (3) for some $\delta \geq 0$, then $z$ fulfills the KKT conditions of (2). This results from the fact that $z \in \Omega \subset \Omega(z, \delta)$ since the active constraints of $\Omega(z, \delta)$ at $z$ are the same as the active constraints of $\Omega$ at $z$.

Given $z \in \Omega$, we define

$$
g_{P}(z)=P_{\Omega}(z-\nabla f(z))-z
$$

We also define

$$
g_{P}(z, \delta)=P_{\Omega(z, \delta)}(z-\nabla f(z))-z
$$

and, for all $\sigma>0$,

$$
g_{P}(z, \delta, \sigma)=P_{\Omega(z, \delta)}(z-\sigma \nabla f(z))-z .
$$

The following identities hold trivially:

$$
g_{P}(z)=g_{P}(z, \infty)=g_{P}(z, \infty, 1)
$$

and

$$
g_{P}(z, \delta)=g_{P}(z, \delta, 1) .
$$

Note that, given $\delta \in[0, \infty]^{p}, \sigma>0$, the fulfillment of the KKT conditions at $z \in \Omega$ is equivalent to $g_{P}(z, \delta, \sigma)=0$. Moreover, if $\delta_{i}^{k} \geq \delta_{\min }>0$ for all $i=1, \ldots, p, k \in \mathbb{N}, z^{k} \rightarrow z$ and $z$ is a KKT point, we have that $\left\|g_{P}\left(z^{k}, \delta^{k}\right)\right\| \rightarrow 0$. This justifies the use of $\left\|g_{P}\left(z^{k}, \delta^{k}\right)\right\|$ as stopping criterion for numerical algorithms.

Finally, if $z \in F_{I} \subset \Omega$, we define

$$
g_{S}(z)=P_{S_{I}}(-\nabla f(z))
$$

\section{Main Algorithm}

Algorithm 3.1 describes the main method presented in this paper to solve linearly constrained optimization problems. All the iterates $x^{k}$ will be feasible if $k \geq 1$. If $x^{0}$ is infeasible, we project this point onto the feasible region for obtaining $x^{1}$. If this projection does not exist, the original problem is infeasible.

At every iteration, $x^{k}$ belongs to a face $F_{I}$. If the norm of the inner gradient $g_{S}\left(x^{k}\right)$ is larger than a tolerance $\varepsilon$, the algorithm judges that it is still worthwhile to stay in the same face and, so, an internal iteration is performed using, essentially, an unconstrained algorithm. In this process, the new iterate may hit the boundary of $F_{I}$. If this happens 20 consecutive times, the algorithm imposes that the next iteration must be of PSPG type. In this way we aim to incorporate as many new constraits as possible.

A PSPG iteration is also performed when $\left\|g_{S}\left(x^{k}\right)\right\|_{\infty} \leq \varepsilon$. By means of PSPG steps we aim to add or remove many constraints at a single iteration. The tolerance vector $\delta^{k}$ for PSPG is chosen adaptively with the only requirement that all the components must be not smaller than 
a fixed parameter $\delta_{\min }>0$. PSPG iterations are more expensive the bigger is $\delta^{k}$ but, on the other hand, very small values of $\delta^{k}$ provide poor information on the geometry of the feasible set.

Algorithm 3.1. (Main Algorithm)

Assume that $x^{0} \in \mathbb{R}^{n}, 0<\sigma_{\min }<\sigma_{\max }<\infty, \alpha \in(0,1 / 2], \delta_{\min }>0, \varepsilon>0$.

Step 1. Compute, if possible, $x^{1}=P_{\Omega}\left(x^{0}\right)$. If this projection does not exist, stop declaring that the original problem is infeasible. Else, set $k \leftarrow 1, k_{1} \leftarrow 0$.

Step 2. If $k_{1}=20$, perform Step 6 .

Step 3. Let $I \subset\{1, \ldots, p\}$ be such that $x^{k} \in F_{I}$. If $\left\|g_{S}\left(x^{k}\right)\right\|_{\infty}>\varepsilon$, perform Steps 4 and 5 , else perform Step 6.

Step 4. Compute $x^{k+1} \in \bar{F}_{I}$ using the Internal Algorithm, whose main characteristics are given in Assumption A1 below.

Step 5. Let $J$ be such that $x^{k+1} \in F_{J}$. If $I$ is strictly contained in $J$, set $k_{1} \leftarrow k_{1}+1$, else set $k_{1} \leftarrow 0$. Set $k \leftarrow k+1$ and go to Step 2 .

Step 6. Choose $\delta^{k} \in \mathbb{R}^{p}$ such that $\delta_{i}^{k} \geq \delta_{\min }$ for all $i=1, \ldots, p$. If $\left\|g_{P}\left(x^{k}, \delta^{k}\right)\right\|_{\infty} \leq \varepsilon$, stop. Else, compute $x^{k+1}$ using a Partial SPG (PSPG) iteration (Algorithm 3.2 below), set $k \leftarrow k+1, k_{1} \leftarrow 0$ and go to Step 3 .

The PSPG iteration used at Step 6 of Algorithm 3.1 is described below.

\section{Algorithm 3.2 (Partial SPG Iteration)}

Step 1. If $k=0$ or $\left(x^{k}-x^{k-1}\right)^{T}\left(\nabla f\left(x^{k}\right)-\nabla f\left(x^{k-1}\right)\right) \leq 0$, set $\sigma_{k}=1$. Else, define $\sigma_{k}$ as the safeguarded spectral coefficient:

$$
\sigma_{k}=\max \left\{\sigma_{\min }, \min \left\{\sigma_{\max }, \frac{\left\|x^{k}-x^{k-1}\right\|^{2}}{\left(x^{k}-x^{k-1}\right)^{T}\left(\nabla f\left(x^{k}\right)-\nabla f\left(x^{k-1}\right)\right)}\right\}\right\} .
$$

Step 2. Define $d^{k}=g_{P}\left(x^{k}, \delta^{k}, \sigma_{k}\right)$.

Step 3. Compute

$$
t_{\text {break }}=\max \left\{t \in[0,1] \mid\left[x^{k}, x^{k}+t d^{k}\right] \subset \Omega\right\} .
$$

Set $t \leftarrow t_{\text {break }}$.

Step 4. Test the Armijo condition

$$
f\left(x^{k}+t d^{k}\right) \leq f\left(x^{k}\right)+\alpha t\left(d^{k}\right)^{T} \nabla f\left(x^{k}\right) .
$$

Step 5. If (4) holds, set $t_{k}=t$, define $x^{k+1}=x^{k}+t_{k} d$ and return. Else, choose

$$
t_{\text {new }} \in[0.1 t, 0.9 t]
$$


set $t \leftarrow t_{\text {new }}$ and go to Step 4 .

The internal steps used at Step 4 of Algorithm 3.1 admit many possible implementations. Essentially, they correspond to the iterations of some convergent algorithm for unconstrained minimization with some modification that corresponds to the case in which the algorithm generates nonfeasible points. Before giving more detailed descriptions we will state here the essential assumption that the Internal Algorithm must satisfy.

\section{Assumption A1}

- If $x^{k+1}$ is computed by the Internal Algorithm and $x^{k} \in F_{I}$, then $x^{k+1} \in \bar{F}_{I}$ and $f\left(x^{k+1}\right) \leq$ $f\left(x^{k}\right)$.

- If $x^{k+j} \in F_{I}$ is computed by the Internal Algorithm for all $j \geq 1$, there exists $j$ such that $\left\|g_{S}\left(x^{k+j}\right)\right\|_{\infty} \leq \varepsilon$.

Taking a basis of the subspace $S_{I}$ and using the coordinates that corresponds to this basis, the auxiliary problem that consists of minimizing $f$ subject to $\bar{F}_{I}$ may be transformed into a minimization problem on a closed convex set $A$. Except in the trivial case in which $F_{I}$ is a single point, $A$ is open and corresponds to the transformation of $F_{I}$. With some abuse of notation, let us express the new equivalent auxiliary problem as:

Minimize $f(x)$ subject to $x \in \bar{A}$.

Assume that Algorithm $U$ is a monotone method for unconstrained optimization with the property that every limit point of a sequence generated by this method is stationary. Then, a typical iteration of a general internal algorithm that satisfies Assumption A1 may be described as follows.

Algorithm 3.3. (Internal Algorithm Iteration)

Step 1. Compute $x^{k+1}$ using Algorithm U. If $x^{k+1} \in A$, return. Else, define $d^{k}=x^{k+1}-x^{k}$ and continue.

Step 2. Compute

$$
t_{\text {break }}=\max \left\{t>0 \mid\left[x^{k}, x^{k}+t d^{k}\right] \subset \bar{A}\right\} .
$$

If $f\left(x^{k}+t_{\text {break }} d^{k}\right)<f\left(x^{k}\right)$, re-define $x^{k+1}=x^{k}+t_{\text {break }} d^{k}$ and return.

Step 3. If

$$
\left(d^{k}\right)^{T} \nabla f\left(x^{k}\right) \geq-10^{-6}\left\|d^{k}\right\|\left\|\nabla f\left(x^{k}\right)\right\|
$$

re-define $d^{k} \leftarrow-\nabla f\left(x^{k}\right)$ and, consequently, re-define $t_{\text {break }}$ as in (6).

Step 4. Set $t \leftarrow t_{\text {break }}$.

Step 5. Test the Armijo inequality

$$
f\left(x^{k}+t d^{k}\right) \leq f\left(x^{k}\right)+\alpha t\left(d^{k}\right)^{T} \nabla f\left(x^{k}\right) .
$$


Step 6. If (7) is fulfilled, define $t_{k}=t, x^{k+1}=x^{k}+t_{k} d^{k}$ and return. Otherwise, choose $t_{\text {new }} \in[0.1 t, 0.9 t]$, set $t \leftarrow t_{\text {new }}$ and go to Step 5 .

Theorem 3.1. Algorithm 3.1 satisfies Assumption A1.

Proof. By construction, the first requirement of Assumption A1 trivially holds. Let us go to the second requirement. If, for all $k$ large enough, the iterations are computed by Algorithm $\mathrm{U}$, the desired property comes from the basic assumption on this algorithm. It remains to consider the case in which, for infinitely many iterations, one has that $x^{k+1}$ is computed at Step 6 . In this case, using standard arguments on minimization algorithms based on sufficient descent directions, we get that any limit point of the corresponding subsequence is, in fact, stationary. Therefore, the theorem is proved.

In our implementations, we used two differents algorithms with the assumption needed by Algorithm U. One is based on the unconstrained procedure implicit in GENCAN [15] and the other on the unconstrained trust-region procedure that underlies BETRA [6]. These methods will be called GenLin and Betralin, respectively.

\section{Convergence}

In this section we prove that Algorithm 3.1 necessarily terminates at a point that satisfies $\left\|g_{P}\left(x^{k}, \delta^{k}\right)\right\|_{\infty} \leq \varepsilon$.

The following auxiliary algorithm is a monotone version of the SPG method $[17,18]$.

Algorithm 4.1. (Monotone SPG)

Let $\widehat{\Omega} \subset \mathbb{R}^{n}$ be a closed and convex set. Assume $\alpha \in(0,1), 0<\sigma_{\min }<\sigma_{\max }<\infty, t_{\min }>0$. Let $x^{0} \in \widehat{\Omega}$ be an arbitrary initial point. Given $x^{k} \in \widehat{\Omega}, \sigma_{k} \in\left[\sigma_{\min }, \sigma_{\max }\right]$, the steps of the $k$-th iteration of the algorithm are:

Step 1. Compute the search direction

Compute

$$
d^{k}=P_{\widehat{\Omega}}\left(x^{k}-\sigma_{k} \nabla f\left(x^{k}\right)\right)-x^{k} .
$$

If $d^{k}=0$, stop the execution of the algorithm declaring that $x_{k}$ is a stationary point.

Step 2. Compute the steplength

Set $t_{\text {ini }} \geq t_{\min }$ and $t=t_{\text {ini }}$. If

$$
f\left(x^{k}+t d^{k}\right) \leq f\left(x^{k}\right)+\alpha t \nabla f\left(x^{k}\right)^{T} d^{k},
$$

set $x^{k+1}$ such that $f\left(x^{k+1}\right) \leq f\left(x^{k}+t d^{k}\right)$ and finish the iteration. Otherwise, choose $t_{\text {new }} \in$ $[0.1 t, 0.9 t]$, set $t \leftarrow t_{\text {new }}$ and repeat test (8). 
Theorem 4.1. Let $\widehat{\Omega}$ be convex and closed. Assume that the sequence generated by Algorithm 4.1 is bounded.Then:

1. If Algorithm 4.1 does not terminate at $x^{k} \in \widehat{\Omega}$, then $x^{k+1}$ is well defined.

2. If Algorithm 4.1 terminates at $x^{k}$ then $x^{k}$ is a stationary point of the problem

$$
\text { Minimize } f(x) \text { subject to } x \in \widehat{\Omega} \text {. }
$$

3. If $x^{*}$ is a limit point of a sequence generated by Algorithm 4.1 then $x^{*}$ is a KKT point of (9). Moreover,

$$
\lim _{k \rightarrow \infty}\left\|d^{k}\right\|=\lim _{k \rightarrow \infty}\left\|P_{\widehat{\Omega}}\left(x^{k}-\sigma_{k} \nabla f\left(x^{k}\right)\right)-x^{k}\right\|=\lim _{k \rightarrow \infty}\left\|P_{\widehat{\Omega}}\left(x^{k}-\nabla f\left(x^{k}\right)\right)-x^{k}\right\|=0 .
$$

Proof. See, for example, Theorem 2.1 of [19].

Now we are in position of proving the convergence of Algorithm 3.1.

Theorem 4.2. Assume that Algorithm 3.1 is applied to problem (2). Let $\Omega$ be bounded. Then:

1. For all $k=0,1,2, \ldots$, if the algorithm does not terminate at $x^{k}$, then $x^{k+1}$ is well defined.

2. The sequence $\left\{x^{k}\right\}$ generated by the algorithm terminates in a finite number of iterations at a point where $\left\|g_{P}\left(x^{k}, \delta^{k}\right)\right\|_{\infty} \leq \varepsilon$.

Proof. The first part of the thesis follows from Assumption A1 and Theorem 4.1. Let us prove the second part. Assume, by contradiction, that the algorithm generates infinitely many iterations. We consider two cases.

1. There are infinitely many iterations of PSPG type.

2. There are only finitely many iterations of PSPG type.

Consider the first case. Let $K \subset \mathbb{N}$ be the set of indices $k$ such that $x^{k+1}$ is computed by the Partial SPG method. Since the number of faces $F_{I}$ is finite, there exists $I \subset\{1, \ldots, p\}$ and $K_{1}$ an infinite subset of $K$ such that $x^{k} \in F_{I}$ for all $k \in K_{1}$. Now, the number of different subsets $\Omega\left(x^{k}, \delta^{k}\right)$ is also finite, therefore, there exist $\widehat{\Omega}$ and $K_{2}$, an infinite subset of $K_{1}$, such that

$$
\Omega\left(x^{k}, \delta^{k}\right)=\widehat{\Omega} \text { for all } k \in K_{2} .
$$

Therefore, for all $k \in K_{2}$,

$$
d^{k}=P_{\widehat{\Omega}}\left(x^{k}-\sigma_{k} \nabla f\left(x^{k}\right)\right)-x^{k} .
$$

Assume, without loss of generality, that $\widehat{\Omega}$ is defined by the inequalities

$$
\left(a^{i}\right)^{T} x-b_{i} \leq 0 \text { for all } i=1, \ldots, q .
$$

Let $i \in\{q+1, \ldots, p\}$. Then,

$$
\left(a^{i}\right)^{T} x^{k}-b_{i}<-\delta_{i}^{k}
$$


Assume that $t \geq 0$ is such that

$$
\left(a^{i}\right)^{T}\left(x^{k}+t d^{k}\right)-b_{i}=0 .
$$

Thus,

Therefore,

$$
t=\frac{b_{i}-\left(a^{i}\right)^{T} x^{k}}{\left(a^{i}\right)^{T} d^{k}}
$$

$$
t>\frac{\delta_{i}^{k}}{\left\|a^{i}\right\|\left\|d^{k}\right\|}
$$

Since $\Omega$ is compact, the sequence $\left\{x^{k}\right\}$ is bounded and, so, the sequence $\left\{d^{k}\right\}$ is bounded too. Say, $\left\|d^{k}\right\| \leq c$ for all $k$. Therefore,

$$
t>\frac{\delta_{i}^{k}}{\left\|a^{i}\right\| c} \geq \frac{\min \left\{\delta_{i}^{k}\right\}}{\max \left\{\left\|a^{i}\right\|\right\} c} \equiv t_{\text {min }} .
$$

This implies that the first trial point of the form $x^{k}+t_{\text {break }} d^{k}$ that is tested in the PSPG algorithm is bounded away from $t_{\min }>0$. Therefore, since $f\left(x^{k+1}\right)<f\left(x^{k}\right)$ for all $k$, the sequence $\left\{x^{k}\right\}_{k \in K_{2}}$ may be thought, after relabeling, as being generated by Algorithm 4.1 applied to the minimization of $f$ on $\widehat{\Omega}$ (taking $t_{\text {ini }}=t_{\text {break }}$ ). Therefore, any limit point $x^{*}$ of this sequence is a stationary point of the auxiliary problem. Since $\Omega \subset \widehat{\Omega}$ this implies that $x^{*}$ is a stationary point of (2). So, $\lim _{k \rightarrow K_{2}}\left\|g_{P}\left(x^{k}, \delta^{k}\right)\right\|=0$. Therefore, for $k \in K_{2}$ large enough, we have that $\left\|g_{P}\left(x^{k}, \delta^{k}\right)\right\|_{\infty} \leq \varepsilon$ and the algorithm would have stopped at $x^{k}$. This contradicts the assumption that $\left\{x^{k}\right\}$ is infinite.

In the case that there are only finitely many iterations of PSPG type, we have that, for $k$ large enough, all the iterations are internal. Moreover, after a finite number of iterations, all the iterates belong to the same face $F_{I}$. By Assumption A1, this implies that there exists $k$ such that $\left\|g_{S}\left(x^{k}\right)\right\|_{\infty} \leq \varepsilon$. Therefore, at this iteration we must have that $\left\|g_{P}\left(x^{k}, \delta^{k}\right)\right\|_{\infty} \leq \varepsilon$, otherwise the next iterate would have been obtained by PSPG.

\section{Implementation}

For the implementation of Algorithm 3.1 several decisions are necessary. The practical efficiency of the algorithm strongly depends on the correctness of them. We will sketch the main algorithmic decisions in this section.

\subsection{Projection Algorithm}

Algorithm 3.1 computes projections on the polytopes $\Omega$ or $\Omega\left(x^{k}, \delta^{k}\right)$. We adopted Subroutine QL, the Goldfarb-Idnani method [36] implemented by Powell [50] and modified by Schittkowski [57] for computing these projections. Subroutine QL can be downloaded from the web site (www.scilab.org) of the open source software Scilab (scientific software package for numerical computations). The Goldfarb-Idnani algorithm is a dual method for positive definite quadratic programming that uses the unconstrained minimizer as initial point. It uses Cholesky and QR decompositions as well as low-rank updating of factorizations.

Subroutine QL has two parameters related to stopping criteria: a small tolerance $\varepsilon_{\mathrm{QL}}>0$ and a maximum number of iterations MAXIT $_{\mathrm{QL}}$. On return, there is an output parameter $\mathrm{INFO}_{\mathrm{QL}}$ whose meaning is: 
info $_{\mathrm{QL}}=\mathbf{0}$ : Success. Maximal violation of the normalized constraints (using $1 /\left\|a^{i}\right\|_{\infty}$ for constraint $\left.C_{i}(x)=\left(a^{i}\right)^{T} x-b_{i} \leq 0, i=1, \ldots, p\right)$ smaller than or equal to $\varepsilon_{\mathrm{QL}}$.

info $_{\mathrm{QL}}=\mathbf{1}$ : Maximum number of iterations $\mathrm{MAXIT}_{\mathrm{QL}}$ reached.

info $_{\mathrm{QL}}=\mathbf{2}$ : Accuracy is insufficient to mantain increasing function values.

info $_{\mathrm{QL}}<\mathbf{0}$ : Problem is infeasible.

Note that our Algorithm 3.1 is not necesarily dealing with normalized constraints. Therefore, independently of the stopping criteria satisfied by Subroutine QL, we check feasibility at its final iterate.

Our projections aim to obtain feasible points with tolerance $\varepsilon_{\text {feas }}$. In the terms of $(2)$ this means that

$$
C_{i}(x) \leq \varepsilon_{\text {feas }} \text { for all } i=1, \ldots, p .
$$

In order achieve (10), we proceed as follows:

Step 1. First trial.

Call Subroutine QL with $\varepsilon_{\mathrm{QL}}=\left(\varepsilon_{\text {feas }}\right)^{1.25}$. Let $\bar{x}$ be the projected point and $\mathrm{INFO}_{\mathrm{QL}}$ be the output flag. Let $R=\{\bar{x}\}$. If (10) holds for $\bar{x}$ then return $\bar{x}$ declaring "Projection successfully computed". Otherwise, if $\mathrm{INFO}_{\mathrm{QL}}=0$ then go to Step 2, else go to Step 3.

Step 2. Try tighter tolerances aiming to satisfy (10).

Step 2.1. Set $\varepsilon_{\mathrm{QL}} \leftarrow \varepsilon_{\mathrm{QL}} / 100$.

Step 2.2. Call Subroutine QL with $\varepsilon_{\mathrm{QL}}$. Let $\bar{x}$ be the projected point and $\mathrm{INFO}_{\mathrm{QL}}$ be the output flag. Let $R=R \cup\{\bar{x}\}$. If (10) holds for $\bar{x}$ then return $\bar{x}$ declaring "Projection successfully computed".

Step 2.3. If $\mathrm{INFO}_{\mathrm{QL}}=0$ and $\varepsilon_{\mathrm{QL}}>10^{-16}$ go to Step 2. Otherwise, go to Step 4 .

Step 3. Try a looser tolerance.

Call Subroutine QL with $\varepsilon_{\mathrm{QL}}=\sqrt{\left(\varepsilon_{\mathrm{feas}}\right)^{1.25}}$. Let $\bar{x}$ be the projected point and $\mathrm{INFO}_{\mathrm{QL}}$ be the output flag. Let $R=R \cup\{\bar{x}\}$. If (10) holds for $\bar{x}$ then return $\bar{x}$ declaring "Projection successfully computed".

Step 4. Treatment for loss of feasibility.

Let $\hat{x}=\operatorname{argmin}_{x \in R}\left\{\max _{i=1, \ldots, p}\left\{C_{i}(x)\right\}\right\}$. If $C_{i}(\hat{x}) \leq \sqrt{\varepsilon_{\text {feas }}}$ for all $i=1, \ldots, p$, then return $\hat{x}$ declaring "Partial loss of feasibility" and continue the execution of the main algorithm. Otherwise, stop the main algorithm declaring "Projection failure". (A second algorithmic option, not used in the experiments reported in this paper, is not to tolerate any loss of feasibility and to stop the main algorithm declaring "Projection failure" when arriving to this step.)

\subsection{Internal Algorithms}

As mentioned in Section 3, we employed two internal methods for computing iterations that preserve the current face $F_{I}$. The first (GENLIN) is a variation of GENCAN [15] and the second 
(BETRALin) is the adaptation of Betra [6] to the case in which the internal face is a polytope, instead of a box (as in [6] and [15]). The "unconstrained directions" of GENLIN are, therefore, inexact-Newton directions preconditioned with the technique presented in [16]. On the other hand, Betralin, as Betra, uses a trust-region strategy. The differences between Genlin and Betralin in terms of robustness and efficiency were not meaningful in our experiments. Therefore, we are going to report here only experiments related to GENLIN.

\subsection{Linear Algebra}

For computing internal iterations one needs to transform the face $F_{I}$ into a convex polytope with nonempty interior in a finite-dimensional Euclidian space. The tool for doing this is to compute a basis of the parallel subspace $S_{I}$. In this way, every point of $F_{I}$ may be expressed as the addition of $x^{k}$ and a linear combination of the coefficients of the basis. The constraints are defined in terms of these coefficients in an obvious way. The basis of $S_{I}$ is computed using the LQ factorization of the matrix formed by the rows $a^{i}, i \in I$. When the face $F_{I}$ changes and new constraints need to be added to form the new current face, the corresponding LQ factorization is updated using the technique described in [35].

\subsection{Feasibility and Rounding}

In the absence of rounding errors, all the iterates of Algorithm 3.1 are feasible. As a consequence, the algorithm may stop only when the criterion $\left\|g_{P}\left(x^{k}, \delta^{k}\right)\right\|_{\infty} \leq \varepsilon$ is satisfied, as established by the convergence theory. In floating point computations, however, small losses of feasibility may occur at different stages of the algorithm. The default option in the presence of feasibility loss is to tolerate infeasibilities up to the level $\sqrt{\varepsilon_{\text {feas }}}$ while still requiring projections to satisfy (10). Projections and Partial SPG iterations are used to correct higher levels of infeasibility. As a consequence, at a final successful point $x^{*}$, it may happen that $\varepsilon_{\text {feas }} \leq C_{i}\left(x^{*}\right) \leq \sqrt{\varepsilon_{\text {feas }}}$. In our experiments, although feasibility was partially lost at a few iterations in a few problems, we always recovered the inequalities (10) at the final point.

If, due to rounding errors, the Internal Algorithm fails to satisfy the monotonicity assumption, a Partial SPG iteration is performed to (presumably) correct this anomaly.

\subsection{Parameters and Stopping Criteria}

In Algorithm 3.1 we use $\varepsilon=10^{-8}$ for the stopping criterion related to the sup-norm of $g_{P}\left(x^{k}, \delta^{k}\right)$. We used $\varepsilon_{\text {feas }}=\varepsilon$ in (10). The choice of $\delta^{k}$ will be explained below. We set $\sigma_{\min }=10^{-10}$, $\sigma_{\max }=10^{10}$ (for the safeguarded spectral coefficient of Algorithm 3.2). We use $\alpha=10^{-4}$ for the Armijo condition of Algorithm 3.2. In the implementation of Algorithm 3.3, we use the default parameters of GENCAN and BETRA (see [6] and [15] for details.)

\section{$6 \quad$ Numerical Experiments}

The objective of the present section is to test the reliability of Algorithm 3.1, with the implementation features described in Section 5. We chose, as test problems, all the linearly constrained examples included in the CuTEr collection [20], with a limited size. Above this size, sparse matrix techniques need to be used for factorizations, which are not employed in our present 
implementation. The CUTEr collection has been chosen for testing due to its popularity among algorithmic developers.

We compared GenLIN with the Harwell subroutine VE11 [51], with the popular active set method for large-scale optimization MiNos [49] and with the interior-point algorithm for large nonlinear programming IPOPT [61]. MINOs and IPOPT use sparse matrix technology, which has a benefical effect in the larger problems chosen in this study, therefore, time comparisons with respect to these two algorithms are not meaningful. However, it is interesting to compare the results with respect to the capacity of finding probably global minimizers and the number of function evaluations performed.

All the experiments were run using a 2.2GHz AMD Athlon 64 Processor $3200+$ with $1.0 \mathrm{~GB}$ of RAM memory and a (32 bits) Linux Operating System (Debian 3.4.6-6). The algorithms were coded in double precision Fortran 77 and compiled with g77 (GNU Fortran (GCC) 3.4.6). The compiler optimization option -O3 was adopted.

\subsection{Linearly Constrained Test Problems}

Linearly constrained optimization problems can always be formulated in the form (2). However, in practice, one uses to say that this type of problems possess three types of constraints: bounds on the variables, equality constraints and proper (not bound) inequality constraints. Of course, each equality constraint can be conceptually reduced to two inequality constraints and, for the basic description of our Algorithm 3.1, this distinction is quite irrelevant.

From now on, $n_{\text {bounds }}$ will be the number of bound constraints of a problem. We consider that a variable bounded both above and below contributes with two bounds. We denote by $n_{\text {eq }}$ the number of equality constraints and by $n_{\text {ineq }}$ the number of inequality constraints. The number of independent variables will be denoted by $n$. We selected, for our tests, all the problems of the CUTEr collection with at most 500 variables and with $1 \leq n_{\text {eq }}+n_{\text {ineq }} \leq 2000$. This corresponds to 133 problems. The main characteristics of the problems are in Table 1. In this table, the problems are roughly ordered from the smallest to the largest one. Some problems from CUTEr have variables where the lower bound coincides with the upper bound. In Table $1, n=n_{1}\left(n_{2}\right)$ means that problem has $n_{1}$ genuine variables plus $n_{2}$ fixed variables. In this case, $n_{\text {bounds }}$ is the number of bounds imposed to the genuine variables.

Problems NASh, MOdel, ARglale, ARGlble, ARglCle and LinCONT are infeasible and, thus, Algorithm 3.1 stops at Step 1 saying that it failed to compute $x^{1}$ (the projection of the initial guess $x^{0}$ onto the feasible set). These 6 problems were removed from the experiments below. So, from now on we consider the remaining 127 problems.

\subsection{Supporting Internal Decisions}

\subsubsection{Choice of $\delta^{k}$}

We tried two choices for the Partial SPG parameters $\delta^{k}$ :

Choice 1: $\delta_{i}^{k}=\bar{\delta}^{k} \max \left\{1,\left|b_{i}\right|\right\}$, where $\bar{\delta}^{1}=0.1$ and $\bar{\delta}^{k+1}=100 \bar{\delta}^{k}$ whenever the iteration $k$ is PSPG and $t_{\text {break }}<0.1$. Else $\bar{\delta}^{k+1}=\bar{\delta}^{k}$.

Choice 2: $\delta_{i}^{k} \equiv \infty \forall i$ and $\forall k$.

The second choice corresponds to use the full SPG iteration in the cases in which PSPG is invoked. 


\begin{tabular}{|c|c|c|c|c|c|c|c|c|c|}
\hline Problem & $n$ & $n_{\text {bounds }}$ & $n_{\mathrm{eq}}$ & $n_{\text {ineq }}$ & Problem & $n$ & $n_{\text {bounds }}$ & $n_{\mathrm{eq}}$ & $n_{\text {ineq }}$ \\
\hline EXTRASIM & 2 & 1 & 1 & 0 & AVGASB & 8 & 16 & 0 & 10 \\
\hline HS9 & 2 & 0 & 1 & 0 & DUALC5 & 8 & 16 & 1 & 277 \\
\hline TAME & 2 & 2 & 1 & 0 & DUALC8 & 8 & 16 & 1 & 502 \\
\hline HS21 & 2 & 4 & 0 & 1 & DUALC1 & 9 & 18 & 1 & 214 \\
\hline HS35MOD & $2(1)$ & 2 & 0 & 1 & HS112 & 10 & 10 & 3 & 0 \\
\hline HUBFIT & 2 & 1 & 0 & 1 & ODFITS & 10 & 10 & 6 & 0 \\
\hline LSQFIT & 2 & 1 & 0 & 1 & GENHS28 & 10 & 0 & 8 & 0 \\
\hline BOOTH & 2 & 0 & 2 & 0 & PORTFL1 & 12 & 24 & 1 & 0 \\
\hline HIMMELBA & 2 & 0 & 2 & 0 & PORTFL2 & 12 & 24 & 1 & 0 \\
\hline SUPERSIM & 2 & 1 & 2 & 0 & PORTFL3 & 12 & 24 & 1 & 0 \\
\hline SIMPLLPA & 2 & 2 & 0 & 2 & PORTFL4 & 12 & 24 & 1 & 0 \\
\hline ZECEVIC2 & 2 & 4 & 0 & 2 & PORTFL6 & 12 & 24 & 1 & 0 \\
\hline HS24 & 2 & 2 & 0 & 3 & LOTSCHD & 12 & 12 & 7 & 0 \\
\hline SIMPLLPB & 2 & 2 & 0 & 3 & HS118 & 15 & 30 & 0 & 29 \\
\hline $\mathrm{PT}$ & 2 & 0 & 0 & 501 & HS119 & 16 & 32 & 8 & 0 \\
\hline SIPOW1M & 2 & 0 & 0 & 2000 & NASH & $18(54)$ & 6 & 24 & 0 \\
\hline SIPOW1 & 2 & 0 & 0 & 2000 & FCCU & 19 & 19 & 8 & 0 \\
\hline SIPOW2M & 2 & 0 & 0 & 2000 & RES & 20 & 40 & 12 & 2 \\
\hline SIPOW2 & 2 & 0 & 0 & 2000 & DEGENLPA & 20 & 40 & 15 & 0 \\
\hline HS28 & 3 & 0 & 1 & 0 & DEGENLPB & 20 & 40 & 15 & 0 \\
\hline HS62 & 3 & 6 & 1 & 0 & KSIP & 20 & 0 & 0 & 1001 \\
\hline HS35I & 3 & 6 & 0 & 1 & MAKELA4 & 21 & 0 & 0 & 40 \\
\hline HS35 & 3 & 3 & 0 & 1 & WATER & 31 & 62 & 10 & 0 \\
\hline HS36 & 3 & 6 & 0 & 1 & LOADBAL & 31 & 42 & 11 & 20 \\
\hline HS37 & 3 & 6 & 0 & 2 & MODEL & $42(1500)$ & 84 & 23 & 15 \\
\hline STANCMIN & 3 & 3 & 0 & 2 & HIMMELBJ & $43(2)$ & 43 & 14 & 0 \\
\hline ZANGWIL3 & 3 & 0 & 3 & 0 & DALLASS & 46 & 92 & 31 & 0 \\
\hline TFI2 & 3 & 0 & 0 & 101 & AVION2 & 49 & 98 & 15 & 0 \\
\hline TFI3 & 3 & 0 & 0 & 101 & GOFFIN & 51 & 0 & 0 & 50 \\
\hline OET1 & 3 & 0 & 0 & 1002 & DUAL4 & 75 & 150 & 1 & 0 \\
\hline $\mathrm{HONG}$ & 4 & 8 & 1 & 0 & LINSPANH & $81(16)$ & 162 & 33 & 0 \\
\hline HS41 & 4 & 8 & 1 & 0 & SPANHYD & $81(16)$ & 162 & 33 & 0 \\
\hline LIN & 4 & 8 & 2 & 0 & QPCBLEND & 83 & 83 & 43 & 31 \\
\hline HS76I & 4 & 8 & 0 & 3 & QPNBLEND & 83 & 83 & 43 & 31 \\
\hline HS76 & 4 & 4 & 0 & 3 & DUAL1 & 85 & 170 & 1 & 0 \\
\hline $\mathrm{S} 277-280$ & 4 & 4 & 0 & 4 & DUAL2 & 96 & 192 & 1 & 0 \\
\hline HS44NEW & 4 & 4 & 0 & 6 & HIMMELBI & 100 & 200 & 0 & 12 \\
\hline HS44 & 4 & 4 & 0 & 6 & DUAL3 & 111 & 222 & 1 & 0 \\
\hline BIGGSC4 & 4 & 8 & 0 & 13 & SMBANK & 117 & 234 & 64 & 0 \\
\hline HATFLDH & 4 & 8 & 0 & 13 & QPCBOEI2 & 143 & 197 & 4 & 181 \\
\hline OET3 & 4 & 0 & 0 & 1002 & QPNBOEI2 & 143 & 197 & 4 & 181 \\
\hline SIPOW3 & 4 & 0 & 0 & 2000 & $\mathrm{AGG}$ & 163 & 163 & 36 & 452 \\
\hline SIPOW4 & 4 & 0 & 0 & 2000 & HYDROELS & $167(2)$ & 334 & 0 & 336 \\
\hline HS48 & 5 & 0 & 2 & 0 & GMNCASE1 & 175 & 0 & 0 & 300 \\
\hline HS49 & 5 & 0 & 2 & 0 & GMNCASE4 & 175 & 0 & 0 & 350 \\
\hline BT3 & 5 & 0 & 3 & 0 & GMNCASE2 & 175 & 0 & 0 & 1050 \\
\hline HS50 & 5 & 0 & 3 & 0 & GMNCASE3 & 175 & 0 & 0 & 1050 \\
\hline HS51 & 5 & 0 & 3 & 0 & SSEBLIN & $192(2)$ & 360 & 48 & 24 \\
\hline HS52 & 5 & 0 & 3 & 0 & DALLASM & 196 & 392 & 151 & 0 \\
\hline HS53 & 5 & 10 & 3 & 0 & ARGLCLE & 200 & 0 & 399 & 0 \\
\hline LSNNODOC & 5 & 6 & 4 & 0 & ARGLALE & 200 & 0 & 400 & 0 \\
\hline HS268 & 5 & 0 & 0 & 5 & ARGLBLE & 200 & 0 & 400 & 0 \\
\hline S268 & 5 & 0 & 0 & 5 & PRIMALC1 & 230 & 215 & 0 & 9 \\
\hline HS86 & 5 & 5 & 0 & 10 & PRIMALC2 & 231 & 229 & 0 & 7 \\
\hline EXPFITA & 5 & 0 & 0 & 22 & LINCONT & $249(1008)$ & 0 & 419 & 0 \\
\hline EXPFITB & 5 & 0 & 0 & 102 & PRIMALC5 & 287 & 278 & 0 & 8 \\
\hline EXPFITC & 5 & 0 & 0 & 502 & PRIMAL1 & 325 & 1 & 0 & 85 \\
\hline HS54 & 6 & 12 & 1 & 0 & QPCBOEI1 & 384 & 540 & 9 & 431 \\
\hline HS55 & 6 & 8 & 6 & 0 & QPNBOEI1 & 384 & 540 & 9 & 431 \\
\hline PENTAGON & 6 & 0 & 0 & 15 & QPCSTAIR & $385(82)$ & 385 & 209 & 147 \\
\hline HS21MOD & 7 & 8 & 0 & 1 & QPNSTAIR & $385(82)$ & 385 & 209 & 147 \\
\hline EQC & $7(2)$ & 14 & 0 & 3 & STEENBRA & 432 & 432 & 108 & 0 \\
\hline QCNEW & $7(2)$ & 14 & 0 & 3 & STATIC3 & 434 & 144 & 96 & 0 \\
\hline $\mathrm{QC}$ & $7(2)$ & 14 & 0 & 4 & STEENBRB & 468 & 468 & 108 & 0 \\
\hline DUALC2 & 7 & 14 & 1 & 228 & STEENBRD & 468 & 468 & 108 & 0 \\
\hline HS105 & 8 & 16 & 0 & 1 & STEENBRF & 468 & 468 & 108 & 0 \\
\hline AVGASA & 8 & 16 & 0 & 10 & & & & & \\
\hline
\end{tabular}

Table 1: Linearly constrained selected problems from CuTEr. 
There are 10 problems (BOоTH, HIMMELBA, SUPERSIM, TAME, STANCMIN, ZANGWIL3, HS55, RES, LINSPANH and GMNCASE4) for which the solution is given by $x^{1}$, the projection of the initial point $x^{0}$ onto the feasible set. Since the versions with choices 1 and 2 for $\delta^{k}$ coincide in those cases, we eliminate them from the experiments of the present subsection. So, we have at hand the remaining 117 problems.

Both versions found equivalent functional values at their final iterate in all the 117 problems. (We say that $f_{1}$ and $f_{2}$ are equivalent if

$$
\left.\left[\left|f_{1}-f_{2}\right| \leq \max \left\{10^{-10}, 10^{-6} \min \left\{\left|f_{1}\right|,\left|f_{2}\right|\right\}\right\}\right] \text { or }\left[f_{1} \leq-10^{20} \text { and } f_{2} \leq-10^{20}\right] .\right)
$$

For comparing these two versions of the method we use the CPU time as a performance measure. (Note that the cost of the projection may depend on $\delta^{k}$.)

Figure 1 shows the performance profile [31]. The figure shows that there is no difference in the robustness of both versions. On the other hand, with Choice 1 the method is more efficient. Choice 1 is faster than Choice 2 in $70.08 \%$ of the problems; whereas the percentage of cases in which Choice 2 is faster is $36.75 \%$.

We arrived to the dynamic Choice 1 after tests that showed us that using a fixed small $\delta^{k}$ could be harmful in critical cases. When, in a neighborhood of $x^{k}$ one has many active constraints that are not active at $x^{k}$, small $\delta^{k}-\mathrm{PSPG}$ iterations do not take most of these constraints into account and, so, for obtaining a feasible $x^{k+1}$ one needs to use a small fraction of the natural PSPG step $d^{k}$. As a consequence, few constraints are added at each iteration and convergence is very slow. When this phenomenon is detected, Choice 1 increases $\delta^{k}$, in such a way that the (previously not considered) nearly active constraints are now taken into account for the projection. Similar considerations lead to the tolerant quadratic programming strategy of VE11 [51].

\subsubsection{Choice of $\sigma_{k}$}

According to the experiments in the previous subsection, we adopted Choice 1 for $\delta^{k}$. We will now corroborate that, in Algorithm 3.2, using $\sigma_{k}$ as the safeguarded spectral step $\sigma_{\text {spec }}$, where

$$
\sigma_{\text {spec }}= \begin{cases}\frac{\left\|x^{k}-x^{k-1}\right\|^{2}}{\left(x^{k}-x^{k-1}\right)^{T}\left(\nabla f\left(x^{k}\right)-\nabla f\left(x^{k-1}\right)\right)}, & \text { if }\left(x^{k}-x^{k-1}\right)^{T}\left(\nabla f\left(x^{k}\right)-\nabla f\left(x^{k-1}\right)\right)>0, \\ 1, & \text { otherwise }\end{cases}
$$

is better than using $\sigma_{k} \equiv 1$.

Since both choices coincide for problems with linear or constant objective function, we will exclude the 26 problems with this characteristic. Therefore, we have at hand the remaining 101 problems. Both choices also coincide if Algorithm 3.1 never executes Step 6, as is the case for other 40 problems (including the 10 problems for which $x^{1}$ is the solution). Eliminating these 40 problems too, we arrive to a set of 61 problems to be considered for the numerical experiments of the current subsection.

We will use the number of function evaluations to evaluate the efficiency of these methods. Figure 2 shows the corresponding performance profile. With both choices we found equivalent functional values for all the problems but the figure shows that using the spectral step is considerably more efficient.

The detailed performance of GENLIN is given in Tables 2 and 3. In these tables, ITER is the number of iterations, PSPGit is the number of Partial SPG iterations, FCNT is the 


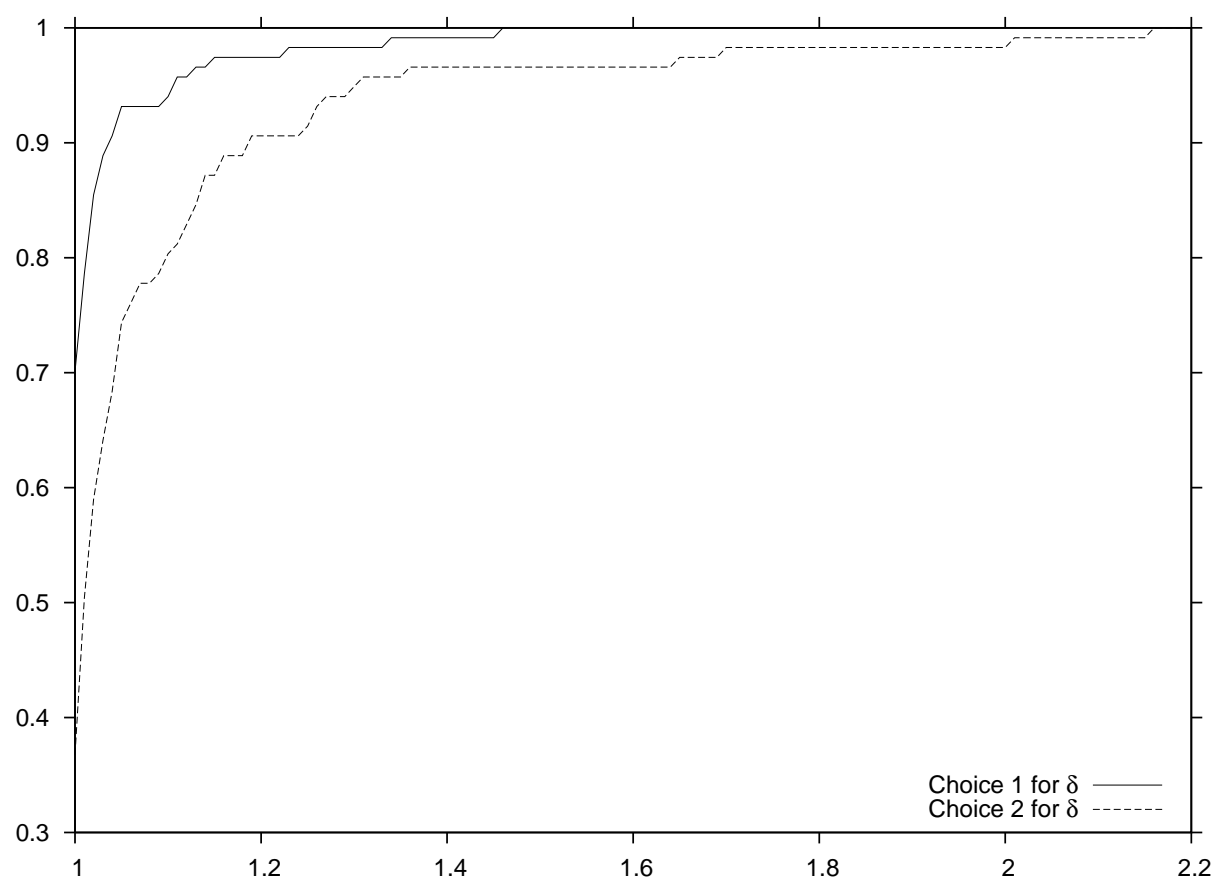

Figure 1: Performance profile comparing choices 1 and 2 of the Partial SPG parameter $\delta^{k}$.

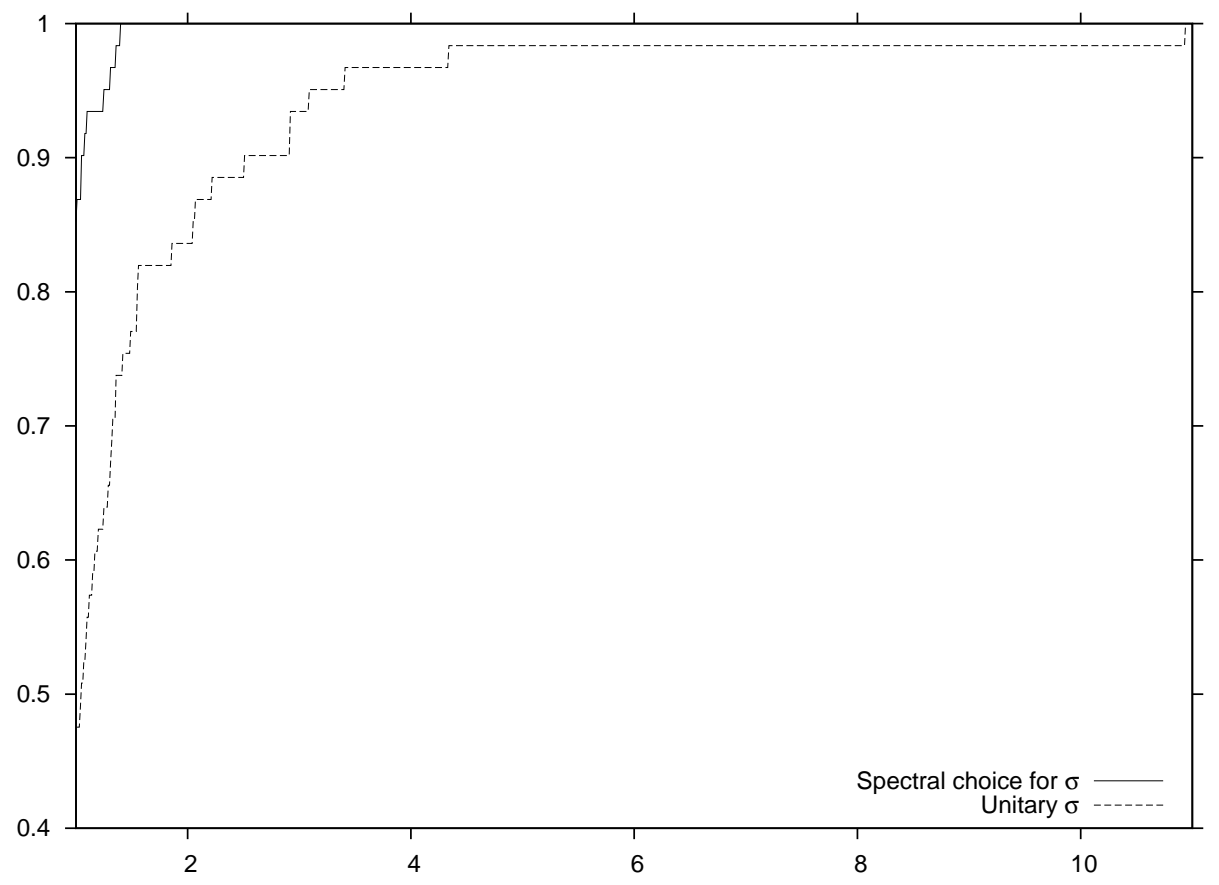

Figure 2: Performance profile comparing the choice of the safeguarded spectral step for $\sigma_{k}$ and $\sigma_{k} \equiv 1$. 


\begin{tabular}{|c|c|c|c|c|c|c|c|c|}
\hline Problem & ITER & PSPGit & FCNT & $f\left(x^{*}\right)$ & $\left\|C\left(x^{*}\right)_{+}\right\|_{\infty}$ & Time & \multicolumn{2}{|c|}{ ProjTime } \\
\hline EXTRASIM & 2 & 0 & 2 & $1.00000 \mathrm{E}+00$ & $4.4 \mathrm{E}-16$ & 0.00007 & $20.70 \%$ & (3) \\
\hline HS9 & 2 & 0 & 6 & $-5.00000 \mathrm{E}-01$ & $1.7 \mathrm{E}-13$ & 0.00007 & $19.30 \%$ & (4) \\
\hline TAME & 1 & 0 & 1 & $0.00000 \mathrm{E}+00$ & $0.0 \mathrm{E}+00$ & $<0.00001$ & $60.00 \%$ & ( 2 ) \\
\hline HS21 & 2 & 0 & 2 & $-9.99600 \mathrm{E}+01$ & $0.0 \mathrm{E}+00$ & 0.00007 & $27.20 \%$ & (4) \\
\hline HS35MOD & 3 & 0 & 3 & $2.50000 \mathrm{E}-01$ & $0.0 \mathrm{E}+00$ & 0.00008 & $8.80 \%$ & (3) \\
\hline HUBFIT & 5 & 1 & 5 & $1.68935 \mathrm{E}-02$ & $0.0 \mathrm{E}+00$ & 0.00018 & $11.10 \%$ & (5) \\
\hline LSQFIT & 5 & 1 & 5 & $3.37870 \mathrm{E}-02$ & $0.0 \mathrm{E}+00$ & 0.00017 & $14.30 \%$ & ( 5$)$ \\
\hline ВOOTH & 1 & 0 & 1 & $0.00000 \mathrm{E}+00$ & $0.0 \mathrm{E}+00$ & $<0.00001$ & $59.00 \%$ & ( 2$)$ \\
\hline HIMMELBA & 1 & 0 & 1 & $0.00000 \mathrm{E}+00$ & $0.0 \mathrm{E}+00$ & $<0.00001$ & $59.60 \%$ & ( 2$)$ \\
\hline SUPERSIM & 1 & 0 & 1 & $6.66667 \mathrm{E}-01$ & $0.0 \mathrm{E}+00$ & $<0.00001$ & $63.30 \%$ & (2) \\
\hline SIMPLLPA & 2 & 1 & 2 & $1.00000 \mathrm{E}+00$ & $0.0 \mathrm{E}+00$ & 0.00007 & $37.50 \%$ & (4) \\
\hline ZECEVIC2 & 3 & 1 & 4 & $-4.12500 \mathrm{E}+00$ & $0.0 \mathrm{E}+00$ & 0.00010 & $17.20 \%$ & (5) \\
\hline HS24 & 3 & 0 & 3 & $-1.00000 \mathrm{E}+00$ & $0.0 \mathrm{E}+00$ & 0.00011 & $3.80 \%$ & ( 3 ) \\
\hline SIMPLLPB & 2 & 0 & 2 & $1.10000 \mathrm{E}+00$ & $0.0 \mathrm{E}+00$ & 0.00007 & $22.80 \%$ & (3) \\
\hline $\mathrm{PT}$ & 4 & 2 & 4 & $1.78394 \mathrm{E}-01$ & $0.0 \mathrm{E}+00$ & 0.00095 & $25.60 \%$ & $(5)$ \\
\hline SIPOW1M & 15 & 9 & 15 & $-1.00000 \mathrm{E}+00$ & $0.0 \mathrm{E}+00$ & 0.01140 & $3.30 \%$ & $(12)$ \\
\hline SIPOW1 & 15 & 9 & 15 & $-1.00000 \mathrm{E}+00$ & $0.0 \mathrm{E}+00$ & 0.01150 & $5.90 \%$ & $(12)$ \\
\hline SIPOW2M & 14 & 8 & 14 & $-1.00000 \mathrm{E}+00$ & $0.0 \mathrm{E}+00$ & 0.01010 & $3.10 \%$ & ( 11$)$ \\
\hline SIPOW2 & 15 & 8 & 15 & $-1.00000 \mathrm{E}+00$ & $0.0 \mathrm{E}+00$ & 0.01080 & $1.10 \%$ & ( 11$)$ \\
\hline HS28 & 2 & 0 & 2 & $3.59918 \mathrm{E}-30$ & $1.6 \mathrm{E}-15$ & 0.00004 & $23.20 \%$ & $(3)$ \\
\hline HS62 & 10 & 0 & 13 & $-2.62725 \mathrm{E}+04$ & $0.0 \mathrm{E}+00$ & 0.00043 & $6.10 \%$ & (4) \\
\hline HS35I & 3 & 0 & 3 & $1.11111 \mathrm{E}-01$ & $0.0 \mathrm{E}+00$ & 0.00013 & $15.80 \%$ & (4) \\
\hline HS35 & 3 & 0 & 3 & $1.11111 \mathrm{E}-01$ & $0.0 \mathrm{E}+00$ & 0.00012 & $4.50 \%$ & (4) \\
\hline HS36 & 4 & 0 & 4 & $-3.30000 \mathrm{E}+03$ & $0.0 \mathrm{E}+00$ & 0.00015 & $14.80 \%$ & (4) \\
\hline HS37 & 6 & 0 & 6 & $-3.45600 \mathrm{E}+03$ & $0.0 \mathrm{E}+00$ & 0.00023 & $14.00 \%$ & (4) \\
\hline STANCMIN & 1 & 0 & 1 & $4.25000 \mathrm{E}+00$ & $0.0 \mathrm{E}+00$ & $<0.00001$ & $73.30 \%$ & ( 2$)$ \\
\hline ZANGWIL3 & 1 & 0 & 1 & $0.00000 \mathrm{E}+00$ & $0.0 \mathrm{E}+00$ & $<0.00001$ & $74.40 \%$ & $(2)$ \\
\hline TFI2 & 22 & 10 & 22 & $6.49031 \mathrm{E}-01$ & $5.6 \mathrm{E}-17$ & 0.00187 & $17.00 \%$ & $(13)$ \\
\hline TFI3 & 5 & 2 & 5 & $4.30116 \mathrm{E}+00$ & $0.0 \mathrm{E}+00$ & 0.00048 & $33.00 \%$ & (6) \\
\hline OET1 & 13 & 6 & 13 & $5.38243 \mathrm{E}-01$ & $2.8 \mathrm{E}-17$ & 0.00667 & $7.30 \%$ & $(9)$ \\
\hline $\mathrm{HONG}$ & 7 & 0 & 7 & $2.25711 \mathrm{E}+01$ & $2.2 \mathrm{E}-16$ & 0.00031 & $20.00 \%$ & $(4)$ \\
\hline HS41 & 6 & 1 & 6 & $1.92593 \mathrm{E}+00$ & $4.4 \mathrm{E}-16$ & 0.00023 & $28.60 \%$ & ( 5$)$ \\
\hline LIN & 2 & 1 & 3 & $-1.75775 \mathrm{E}-02$ & $5.0 \mathrm{E}-13$ & 0.00009 & $45.30 \%$ & ( 5$)$ \\
\hline HS76I & 6 & 1 & 6 & $-4.68182 \mathrm{E}+00$ & $8.9 \mathrm{E}-16$ & 0.00025 & $16.10 \%$ & (5) \\
\hline HS76 & 6 & 1 & 6 & $-4.68182 \mathrm{E}+00$ & $8.9 \mathrm{E}-16$ & 0.00023 & $15.10 \%$ & ( 5$)$ \\
\hline $\mathrm{S} 277-280$ & 4 & 0 & 4 & $5.07619 \mathrm{E}+00$ & $0.0 \mathrm{E}+00$ & 0.00017 & $13.50 \%$ & ( 3 ) \\
\hline HS44NEW & 6 & 0 & 6 & $-1.50000 \mathrm{E}+01$ & $0.0 \mathrm{E}+00$ & 0.00024 & $12.10 \%$ & (3) \\
\hline HS44 & 6 & 2 & 6 & $-1.50000 \mathrm{E}+01$ & $0.0 \mathrm{E}+00$ & 0.00023 & $24.20 \%$ & (5) \\
\hline BIGGSC4 & 4 & 1 & 4 & $-2.43750 \mathrm{E}+01$ & $0.0 \mathrm{E}+00$ & 0.00018 & $17.90 \%$ & (4) \\
\hline HATFLDH & 3 & 1 & 3 & $-2.43750 \mathrm{E}+01$ & $0.0 \mathrm{E}+00$ & 0.00014 & $41.90 \%$ & ( 5$)$ \\
\hline OET3 & 4 & 2 & 4 & $4.50505 \mathrm{E}-03$ & $2.8 \mathrm{E}-17$ & 0.00281 & $37.90 \%$ & $(5)$ \\
\hline SIPOW3 & 9 & 3 & 9 & $5.34659 \mathrm{E}-01$ & $0.0 \mathrm{E}+00$ & 0.00950 & $6.20 \%$ & (6) \\
\hline SIPOW4 & 11 & 3 & 11 & $2.72362 \mathrm{E}-01$ & $5.6 \mathrm{E}-17$ & 0.01260 & $6.70 \%$ & (6) \\
\hline HS48 & 2 & 0 & 2 & $3.94430 \mathrm{E}-31$ & $8.9 \mathrm{E}-16$ & 0.00006 & $25.30 \%$ & ( 3 ) \\
\hline HS49 & 20 & 0 & 20 & $1.06000 \mathrm{E}-11$ & $5.3 \mathrm{E}-15$ & 0.00084 & $4.10 \%$ & (4) \\
\hline BT3 & 2 & 0 & 2 & $4.09302 \mathrm{E}+00$ & $2.8 \mathrm{E}-15$ & 0.00010 & $40.60 \%$ & (4) \\
\hline HS50 & 10 & 0 & 10 & $2.66302 \mathrm{E}-28$ & $2.2 \mathrm{E}-14$ & 0.00039 & $8.50 \%$ & ( 3 ) \\
\hline HS51 & 2 & 0 & 2 & $1.77494 \mathrm{E}-30$ & $1.3 \mathrm{E}-15$ & 0.00006 & $29.20 \%$ & ( 3 ) \\
\hline HS52 & 2 & 0 & 2 & $5.32665 \mathrm{E}+00$ & $3.2 \mathrm{E}-16$ & 0.00010 & $46.80 \%$ & (4) \\
\hline HS53 & 2 & 0 & 2 & $4.09302 \mathrm{E}+00$ & $2.2 \mathrm{E}-16$ & 0.00010 & $44.70 \%$ & (4) \\
\hline LSNNODOC & 7 & 1 & 7 & $1.23112 \mathrm{E}+02$ & $8.9 \mathrm{E}-16$ & 0.00030 & $21.40 \%$ & ( 5$)$ \\
\hline HS268 & 7 & 2 & 20 & $3.63798 \mathrm{E}-12$ & $0.0 \mathrm{E}+00$ & 0.00033 & $10.50 \%$ & $(5)$ \\
\hline $\mathrm{S} 268$ & 7 & 2 & 20 & $3.63798 \mathrm{E}-12$ & $0.0 \mathrm{E}+00$ & 0.00033 & $9.30 \%$ & ( 5$)$ \\
\hline HS86 & 10 & 2 & 10 & $-3.23487 \mathrm{E}+01$ & $8.9 \mathrm{E}-16$ & 0.00053 & $14.00 \%$ & (6) \\
\hline EXPFITA & 19 & 3 & 19 & $1.13661 \mathrm{E}-03$ & $1.4 \mathrm{E}-14$ & 0.00134 & $3.60 \%$ & ( 7 ) \\
\hline EXPFITB & 24 & 4 & 24 & $5.01937 \mathrm{E}-03$ & $3.8 \mathrm{E}-15$ & 0.00426 & $3.90 \%$ & ( 8$)$ \\
\hline EXPFITC & 46 & 12 & 48 & $2.33026 \mathrm{E}-02$ & $5.7 \mathrm{E}-14$ & 0.03330 & $2.90 \%$ & ( 16$)$ \\
\hline HS54 & 851 & 16 & 861 & $-9.03490 \mathrm{E}-01$ & $1.1 \mathrm{E}-09$ & 0.03920 & $2.00 \%$ & $(20)$ \\
\hline HS55 & 1 & 0 & 1 & $6.66667 \mathrm{E}+00$ & $8.9 \mathrm{E}-16$ & 0.00002 & $78.80 \%$ & $(2)$ \\
\hline PENTAGON & 15 & 0 & 15 & $1.36522 \mathrm{E}-04$ & $0.0 \mathrm{E}+00$ & 0.00073 & $9.10 \%$ & (4) \\
\hline HS21MOD & 2 & 0 & 2 & $-9.59600 \mathrm{E}+01$ & $0.0 \mathrm{E}+00$ & 0.00009 & $47.60 \%$ & (4) \\
\hline EQC & 2 & 1 & 2 & $-8.29548 \mathrm{E}+02$ & $0.0 \mathrm{E}+00$ & 0.00011 & $62.50 \%$ & (4) \\
\hline QCNEW & 2 & 1 & 2 & $-8.06522 \mathrm{E}+02$ & $0.0 \mathrm{E}+00$ & 0.00012 & $66.70 \%$ & (4) \\
\hline $\mathrm{QC}$ & 7 & 0 & 7 & $-9.56538 \mathrm{E}+02$ & $0.0 \mathrm{E}+00$ & 0.00041 & $14.40 \%$ & ( 3$)$ \\
\hline DUALC2 & 7 & 1 & 7 & $3.55131 \mathrm{E}+03$ & $0.0 \mathrm{E}+00$ & 0.00161 & $9.90 \%$ & $(5)$ \\
\hline HS105 & 24 & 2 & 30 & $1.06188 \mathrm{E}+03$ & $0.0 \mathrm{E}+00$ & 0.02570 & $0.60 \%$ & ( 6$)$ \\
\hline AVGASA & 4 & 0 & 4 & $-4.63199 \mathrm{E}+00$ & $1.1 \mathrm{E}-16$ & 0.00024 & $36.70 \%$ & (4) \\
\hline
\end{tabular}

Table 2: Performance of Genlin (Part I). 


\begin{tabular}{|c|c|c|c|c|c|c|c|c|}
\hline Problem & ITER & PSPGit & FCNT & $f\left(x^{*}\right)$ & $\left\|C\left(x^{*}\right)+\right\|_{\infty}$ & Time & \multicolumn{2}{|c|}{ ProjTime } \\
\hline AVGASB & 4 & 0 & 4 & $-4.48322 \mathrm{E}+00$ & $1.2 \mathrm{E}-16$ & 0.00023 & $32.40 \%$ & $(4)$ \\
\hline DUALC5 & 5 & 0 & 5 & $4.27233 \mathrm{E}+02$ & $0.0 \mathrm{E}+00$ & 0.00166 & $6.00 \%$ & (4) \\
\hline DUALC8 & 11 & 2 & 12 & $1.83094 \mathrm{E}+04$ & $0.0 \mathrm{E}+00$ & 0.00571 & $1.90 \%$ & ( 6$)$ \\
\hline DUALC1 & 12 & 2 & 13 & $6.15525 \mathrm{E}+03$ & $0.0 \mathrm{E}+00$ & 0.00323 & $2.50 \%$ & ( 6$)$ \\
\hline HS112 & 22 & 2 & 31 & $-4.77611 \mathrm{E}+01$ & $4.4 \mathrm{E}-16$ & 0.00180 & $7.70 \%$ & ( 6$)$ \\
\hline ODFITS & 6 & 0 & 6 & $-2.38003 \mathrm{E}+03$ & $2.3 \mathrm{E}-13$ & 0.00043 & $35.90 \%$ & (4) \\
\hline GENHS28 & 2 & 0 & 2 & $9.27174 \mathrm{E}-01$ & $1.6 \mathrm{E}-15$ & 0.00020 & $62.90 \%$ & (4) \\
\hline PORTFL1 & 8 & 0 & 8 & $2.04863 \mathrm{E}-02$ & $3.3 \mathrm{E}-16$ & 0.00082 & $12.50 \%$ & (4) \\
\hline PORTFL2 & 9 & 0 & 9 & $2.96892 \mathrm{E}-02$ & $2.2 \mathrm{E}-16$ & 0.00090 & $10.40 \%$ & (4) \\
\hline PORTFL3 & 11 & 1 & 12 & $3.27497 \mathrm{E}-02$ & $2.2 \mathrm{E}-16$ & 0.00107 & $8.20 \%$ & ( 5$)$ \\
\hline PORTFL4 & 8 & 0 & 8 & $2.63070 \mathrm{E}-02$ & $2.2 \mathrm{E}-16$ & 0.00085 & $11.40 \%$ & (4) \\
\hline PORTFL6 & 8 & 0 & 8 & $2.57918 \mathrm{E}-02$ & $1.1 \mathrm{E}-16$ & 0.00084 & $9.10 \%$ & (4) \\
\hline LOTSCHD & 5 & 0 & 5 & $2.39842 \mathrm{E}+03$ & $2.5 \mathrm{E}-14$ & 0.00040 & $57.50 \%$ & (4) \\
\hline HS118 & 16 & 1 & 16 & $6.64820 \mathrm{E}+02$ & $1.4 \mathrm{E}-14$ & 0.00136 & $31.30 \%$ & (5) \\
\hline HS119 & 12 & 1 & 12 & $2.44900 \mathrm{E}+02$ & $8.9 \mathrm{E}-16$ & 0.00195 & $29.90 \%$ & (5) \\
\hline $\mathrm{FCCU}$ & 6 & 1 & 6 & $1.11491 \mathrm{E}+01$ & $8.9 \mathrm{E}-15$ & 0.00069 & $62.30 \%$ & ( 5$)$ \\
\hline RES & 1 & 0 & 1 & $0.00000 \mathrm{E}+00$ & $9.2 \mathrm{E}-15$ & 0.00006 & $87.60 \%$ & $(2)$ \\
\hline DEGENLPA & 3 & 1 & 3 & $3.06039 \mathrm{E}+00$ & $1.1 \mathrm{E}-16$ & 0.00096 & $87.00 \%$ & (4) \\
\hline DEGENLPB & 2 & 0 & 2 & $-3.07312 \mathrm{E}+01$ & $7.5 \mathrm{E}-16$ & 0.00070 & $89.00 \%$ & ( 3 ) \\
\hline KSIP & 23 & 5 & 25 & $5.75798 \mathrm{E}-01$ & $3.3 \mathrm{E}-16$ & 0.07820 & $12.90 \%$ & (9) \\
\hline MAKELA4 & 16 & 0 & 16 & $2.10942 \mathrm{E}-15$ & $3.8 \mathrm{E}-15$ & 0.00149 & $18.20 \%$ & ( 3 ) \\
\hline WATER & 21 & 2 & 23 & $1.05494 \mathrm{E}+04$ & $1.1 \mathrm{E}-13$ & 0.00306 & $65.40 \%$ & ( 6$)$ \\
\hline LOADBAL & 15 & 3 & 14 & $4.52851 \mathrm{E}-01$ & $1.4 \mathrm{E}-14$ & 0.00343 & $47.40 \%$ & $(7)$ \\
\hline HIMMELBJ & 622 & 102 & 1233 & $-1.91034 \mathrm{E}+03$ & $2.0 \mathrm{E}-10$ & 0.22000 & $31.20 \%$ & $(129)$ \\
\hline DALLASS & 49 & 3 & 60 & $-3.23932 \mathrm{E}+04$ & $8.3 \mathrm{E}-14$ & 0.02190 & $33.00 \%$ & $(7)$ \\
\hline AVION2 & 5 & 0 & 5 & $9.46801 \mathrm{E}+07$ & $1.8 \mathrm{E}-12$ & 0.00312 & $78.20 \%$ & (4) \\
\hline GOFFIN & 7 & 0 & 7 & $-8.65974 \mathrm{E}-15$ & $5.4 \mathrm{E}-14$ & 0.00666 & $72.80 \%$ & ( 3 ) \\
\hline DUAL4 & 19 & 1 & 19 & $7.46091 \mathrm{E}-01$ & $0.0 \mathrm{E}+00$ & 0.02750 & $17.00 \%$ & ( 5$)$ \\
\hline LINSPANH & 1 & 0 & 1 & $-7.70000 \mathrm{E}+01$ & $1.4 \mathrm{E}-13$ & 0.00134 & $98.20 \%$ & $(2)$ \\
\hline SPANHYD & 6 & 2 & 7 & $2.39738 \mathrm{E}+02$ & $5.1 \mathrm{E}-13$ & 0.01820 & $90.30 \%$ & ( 6$)$ \\
\hline QPCBLEND & 8 & 2 & 10 & $-7.84254 \mathrm{E}-03$ & $1.2 \mathrm{E}-17$ & 0.03760 & $93.20 \%$ & ( 6$)$ \\
\hline QPNBLEND & 20 & 5 & 22 & $-9.13614 \mathrm{E}-03$ & $1.2 \mathrm{E}-15$ & 0.06240 & $91.30 \%$ & (9) \\
\hline DUAL1 & 49 & 3 & 57 & $3.50130 \mathrm{E}-02$ & $1.1 \mathrm{E}-15$ & 0.13600 & $5.60 \%$ & ( 7 ) \\
\hline DUAL2 & 8 & 0 & 8 & $3.37337 \mathrm{E}-02$ & $8.9 \mathrm{E}-16$ & 0.04150 & $12.40 \%$ & (4) \\
\hline HIMMELBI & 65 & 4 & 67 & $-1.73557 \mathrm{E}+03$ & $2.8 \mathrm{E}-14$ & 0.03680 & $73.70 \%$ & ( 8$)$ \\
\hline DUAL3 & 30 & 3 & 34 & $1.35756 \mathrm{E}-01$ & $5.6 \mathrm{E}-16$ & 0.15500 & $8.30 \%$ & $(7)$ \\
\hline SMBANK & 54 & 5 & 69 & $-7.12929 \mathrm{E}+06$ & $2.3 \mathrm{E}-10$ & 0.18100 & $66.10 \%$ & ( 10$)$ \\
\hline QPCBOEI2 & 10 & 2 & 9 & $8.17196 \mathrm{E}+06$ & $1.8 \mathrm{E}-12$ & 0.17500 & $93.90 \%$ & $(6)$ \\
\hline QPNBOEI2 & 42 & 4 & 40 & $1.37140 \mathrm{E}+06$ & $1.1 \mathrm{E}-12$ & 0.28100 & $87.70 \%$ & ( 8$)$ \\
\hline AGG & 60 & 5 & 60 & $-3.59918 \mathrm{E}+07$ & $4.1 \mathrm{E}-09$ & 0.44900 & $65.30 \%$ & ( 8 ) \\
\hline HYDROELS & 16 & 3 & 16 & $-3.58227 \mathrm{E}+06$ & $3.6 \mathrm{E}-14$ & 0.32900 & $92.00 \%$ & ( 7 ) \\
\hline GMNCASE1 & 16 & 0 & 16 & $2.66973 \mathrm{E}-01$ & $4.2 \mathrm{E}-17$ & 0.25000 & $42.90 \%$ & (4) \\
\hline GMNCASE4 & 1 & 0 & 1 & $5.94688 \mathrm{E}+03$ & $1.8 \mathrm{E}-15$ & 0.23600 & $95.90 \%$ & ( 2$)$ \\
\hline GMNCASE2 & 28 & 1 & 28 & $-9.94445 \mathrm{E}-01$ & $3.5 \mathrm{E}-17$ & 0.64300 & $26.00 \%$ & (5) \\
\hline GMNCASE3 & 26 & 1 & 27 & $1.52515 \mathrm{E}+00$ & $4.2 \mathrm{E}-17$ & 0.63100 & $29.20 \%$ & (5) \\
\hline SSEBLIN & 100 & 4 & 100 & $1.61706 \mathrm{E}+07$ & $5.8 \mathrm{E}-11$ & 0.28600 & $84.10 \%$ & ( 7 ) \\
\hline DALLASM & 40 & 2 & 43 & $-4.81982 \mathrm{E}+04$ & $6.4 \mathrm{E}-13$ & 0.68900 & $73.00 \%$ & ( 6$)$ \\
\hline PRIMALC1 & 6 & 1 & 6 & $-6.15525 \mathrm{E}+03$ & $1.5 \mathrm{E}-11$ & 0.13800 & $100.00 \%$ & ( 5$)$ \\
\hline PRIMALC2 & 5 & 2 & 6 & $-3.55131 \mathrm{E}+03$ & $1.1 \mathrm{E}-10$ & 0.17200 & $100.00 \%$ & ( 6$)$ \\
\hline PRIMALC5 & 7 & 1 & 7 & $-4.27233 \mathrm{E}+02$ & $1.8 \mathrm{E}-11$ & 0.27600 & $97.30 \%$ & ( 5$)$ \\
\hline PRIMAL1 & 3 & 1 & 3 & $-3.50130 \mathrm{E}-02$ & $2.5 \mathrm{E}-16$ & 0.53300 & $98.40 \%$ & ( 5$)$ \\
\hline QPCBOEI1 & 19 & 2 & 20 & $1.15039 \mathrm{E}+07$ & $1.9 \mathrm{E}-13$ & 5.40000 & $94.50 \%$ & ( 6$)$ \\
\hline QPNBOEI1 & 114 & 7 & 119 & $6.77711 \mathrm{E}+06$ & $1.2 \mathrm{E}-12$ & 14.02000 & $91.60 \%$ & $(11)$ \\
\hline QPCSTAIR & 9 & 1 & 9 & $6.20439 \mathrm{E}+06$ & $2.8 \mathrm{E}-14$ & 4.67000 & $97.50 \%$ & $(5)$ \\
\hline QPNSTAIR & 15 & 2 & 15 & $5.14603 \mathrm{E}+06$ & $2.8 \mathrm{E}-14$ & 5.26000 & $96.50 \%$ & ( 6$)$ \\
\hline STEENBRA & 38 & 4 & 37 & $1.69577 \mathrm{E}+04$ & $2.3 \mathrm{E}-13$ & 5.28000 & $98.40 \%$ & ( 8$)$ \\
\hline STATIC3 & 8 & 0 & 8 & $-1.05655 \mathrm{E}+20$ & $2.9 \mathrm{E}-09$ & 2.11000 & $98.90 \%$ & $(7)$ \\
\hline STEENBRB & 116 & 9 & 111 & $9.07586 \mathrm{E}+03$ & $2.3 \mathrm{E}-12$ & 9.96000 & $97.20 \%$ & ( 13$)$ \\
\hline STEENBRD & 144 & 14 & 149 & $9.14472 \mathrm{E}+03$ & $2.3 \mathrm{E}-11$ & 13.83000 & $97.60 \%$ & ( 18$)$ \\
\hline STEENBRF & 114 & 10 & 108 & $8.99185 \mathrm{E}+03$ & $1.5 \mathrm{E}-10$ & 10.76000 & $97.60 \%$ & ( 14$)$ \\
\hline
\end{tabular}

Table 3: Performance of Genlin (Part II). 
number of functional evaluations, $f\left(x^{*}\right)$ is the functional value at the final iterate, $\left\|C\left(x^{*}\right)_{+}\right\|_{\infty}$ is the maximal violation of feasibility, Time is the total CPU time in seconds and ProjTime is the percentage of Time used by Subroutine QL to compute projections (the total number of projections is within parentheses). In the cases in which the CPU time is very small, we obtained the correct value running the subroutine many times and taking the average. In all the problems except StATiC3 and HimmelBJ, GenLin stopped because the criterion $\left\|g_{P}\left(x^{k}, \delta^{k}\right)\right\| \leq \varepsilon=10^{-8}$ was fulfilled. In the case of STATiC3, Genlin stopped because $f\left(x^{k}\right)<-10^{20}$. In the case of HIMMELBJ, GEnLIN stopped at a feasible point with well-defined objetive function, after several trials of evaluating the objective function at iterates where it was not well-defined.

\subsection{Comparison with Ve11}

VE11 is a Harwell subroutine that implements Powell's tolerant method [51]. It has only one relevant parameter $\varepsilon_{\mathrm{VE} 11}$ related to tolerances and stopping criteria, which was defined for these experiments as $\varepsilon_{\mathrm{VE} 11}=10^{-8}$.

We applied VE11 to the 127 feasible problems of Table 1 (details can be found in [7]). The final iterate of VE11 does not satisfy the feasibility requirement (10) with tolerance $\varepsilon_{\text {feas }}=10^{-8}$ in 16 problems:

- Problem steenbra is the unique problem for which VE11 stopped due the CPU time limit of 10 minutes imposed for each pair problem/method in our numerical experiments.

- In problems WATER and STATIC3, VE11 stops saying that the current point is feasible but the line search fails to reduce the objective function value.

- In problems smbank, Dallasm, Steenbrb, Steenbrd, Steenbrf, Ve11 stops saying that the current point is feasible but rounding errors seem to be preventing higher accuracy.

- In problems HS55, LINSPANH and SPANHYD, VE11 stops saying that the equality constraints and the bounds on the variables are incompatible.

- In problems AGG, GMNCASE4, DALlass, QPCBOEI2 and QPNBOEI2, VE11 stops saying that it is possible to satisfy all the equality constraints and bounds but the general inequality constraints cannot be satisfied.

In the other 111 problems, the final iterate of VE11 is feasible (in the sense of (10) with tolerance $\varepsilon_{\text {feas }}=10^{-8}$ ). In 84 out of those 111 problems, VE11 stops satisfying its stopping criterion related to "success". For the remaining 27 problems, the diagnostics provided by the subroutine were:

- In problems HS62 and PRIMALC1, VE11 stops saying the current point is feasible but rounding errors seem to be preventing higher accuracy.

- In problems hS268, s268, QCNEW, DUALC8, hs105, DUALC1, hS119, LOADBAL, AVION2, QPCBLEND, QPNBLEND, DUAl1, DUAL2, DUAL3, hyDROELS, GMNCASE1, GMNCASE2, SSEBLIN, PRIMAL1 and QPCSTAIR, VE11 stops saying that the current point is feasible but the line search fails to reduce the objective function value.

- In problem HimmelBJ, Ve11 stops saying that the equality constraints and the bounds on the variables are incompatible. 
- In problems HIMmelBI, QPCBOEI1, QPNBOEI1 and QPNSTAIR, VE11 stops saying that it is possible to satisfy all the equality constraints and bounds but the general inequality constraints cannot be satisfied.

Considering the 111 problems in which VE11 obtained feasible final points, we observed that, in most of them, the final functional value at the final point is equivalent to that obtained by GenLin. The exceptions were:

1. In 8 problems (LSNNODOC, QPCBLEND, QPNBLEND, HIMMELBI, QPCBOEI1, QPNBOEI1, QPCSTAIR, QPNSTAIR) GENLIN obtained a smaller final functional value than VE11.

2. In 3 problems (HS54, EQC, HS105) VE11 obtained a smaller final functional value than GenLin.

3. In 2 problems (QCNEW, HIMMELBJ) the final functional value provided by VE11 was not defined (NaN message).

In the remaining 98 problems both GENLIN and VE11 obtained feasible points with equivalent functional values. Considering these 98 problems:

1. GenLin performed less function evaluations in 83 cases.

2. VE11 performed less function evaluations in 11 cases.

3. GenLIN and VE11 performed the same number of function evaluations in 4 cases.

4. Genlin was faster than Ve11 (up to a tolerance of 10\%) in 15 cases.

5. VE11 was faster than GenLIN (up to a tolerance of 10\%) in 79 cases.

6. Genlin and VE11 spent the same amount of time (up to a tolerance of 10\%) in 4 cases.

7. Restricting the three items above to the problems in which one of the methods used more than 0.1 seconds (11 problems) GENLIN was faster than VE11 7 times and VE11 was faster than GENLIN 3 times (both methods spent the same time in one case).

A performance profile using the number of functional evaluations as a performance measurement can be seen in Figure 3.

\subsection{Comparison with Minos}

We ran the experiments of this subsection using Minos [49] with feasibility and optimality tolerances $\varepsilon_{\text {feas }}=\varepsilon_{\mathrm{opt}}=10^{-8}$. All the other parameters were set with their default values.

We applied Minos to the 127 feasible problems of Table 1 (details can be found in [7]). The final iterate of MinOs in 2 out of the 127 problems (HS54 and AGG) does not satisfy the feasibility requirement (10) with tolerance $\varepsilon_{\text {feas }}=10^{-8}$. In the other 125 problems, the final iterate of MiNOs is feasible with tolerance $\varepsilon_{\text {feas }}=10^{-8}$. In 119 problems, MinOs stops satisfying its stopping criterion related to "success". For the remaining 6 problems, the diagnostics were:

- In problem Static3, Minos stops declaring that the problem is unbounded (or badly scaled). 


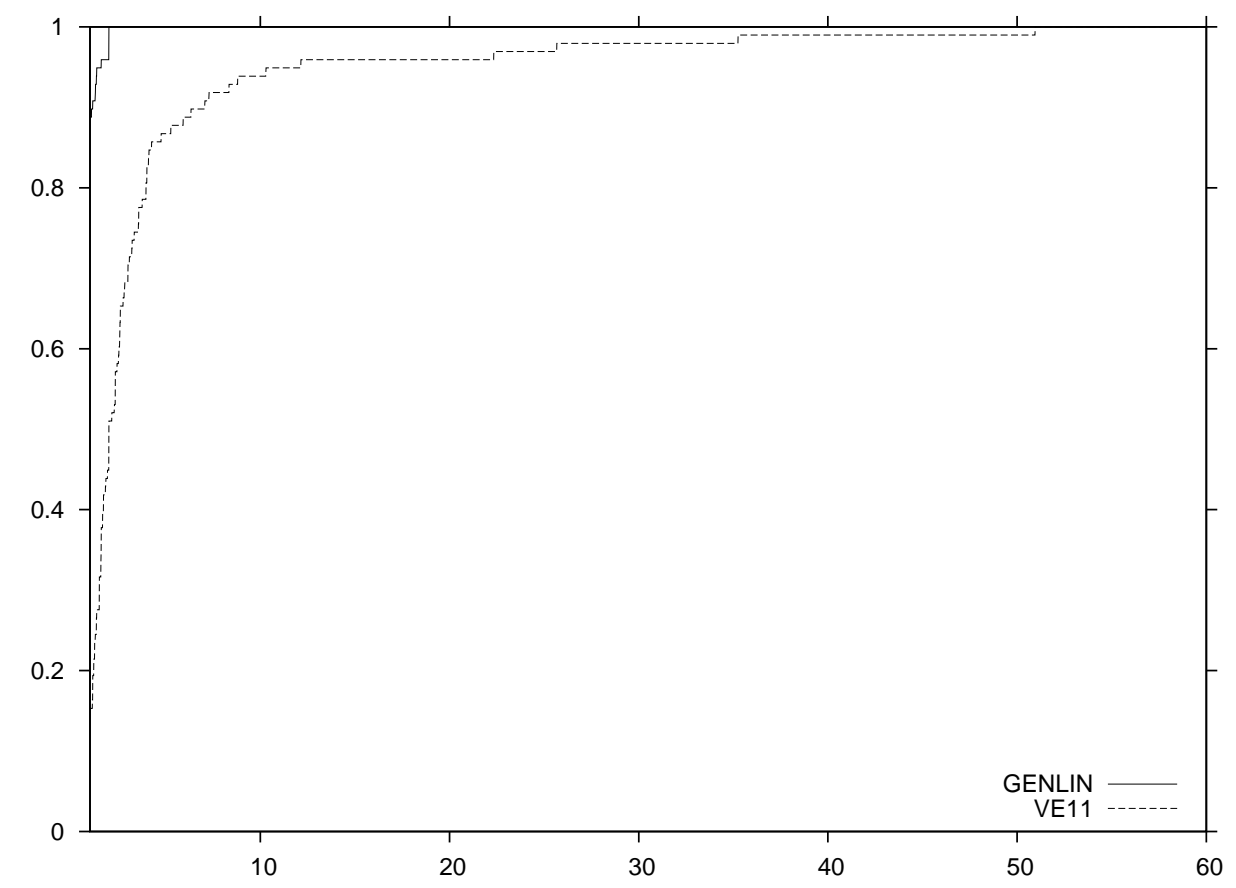

Figure 3: Performance profiles of GENLIN and VE11 in the 83 problems (out of 127) for which both methods obtained feasible points with equivalent functional values. Number of functional evaluations is being used as performance measurement.

- In problem HS55, MiNos stops declaring that the current point cannot be improved.

- In problems hs118, himmelBJ, SPAnhyd and STEenbrd, Minos stops declaring that a near-optimal solution was found.

Considering the 125 problems in which Minos obtained feasible final points, we observed that, in most of them, the functional value at the final point is equivalent to the one obtained by GenLin. The exceptions are:

1. In 6 problems (Hs41, PENTAGOn, QC, HS118, QPNBLEND, STEENBRD) Genlin obtained a smaller functional value than Minos.

2. In 6 problems (hATfldh, Lin, EQC, hs105, DEgenlPA, QPnboei1, Minos obtained a smaller functional value than GENLIN.

3. In one problem (HIMMELBJ) the final functional value provided by Minos was not defined (NaN message).

Considering the 111 problems in which GENLIN and Minos obtained feasible points with equivalent functional values (excluding here problem sTATIC3 for which both methods detected, by different ways, that it seems to be unbounded), we observed that:

1. Genlin performed less function evaluations in 84 cases.

2. Minos performed less function evaluations in 26 cases. 


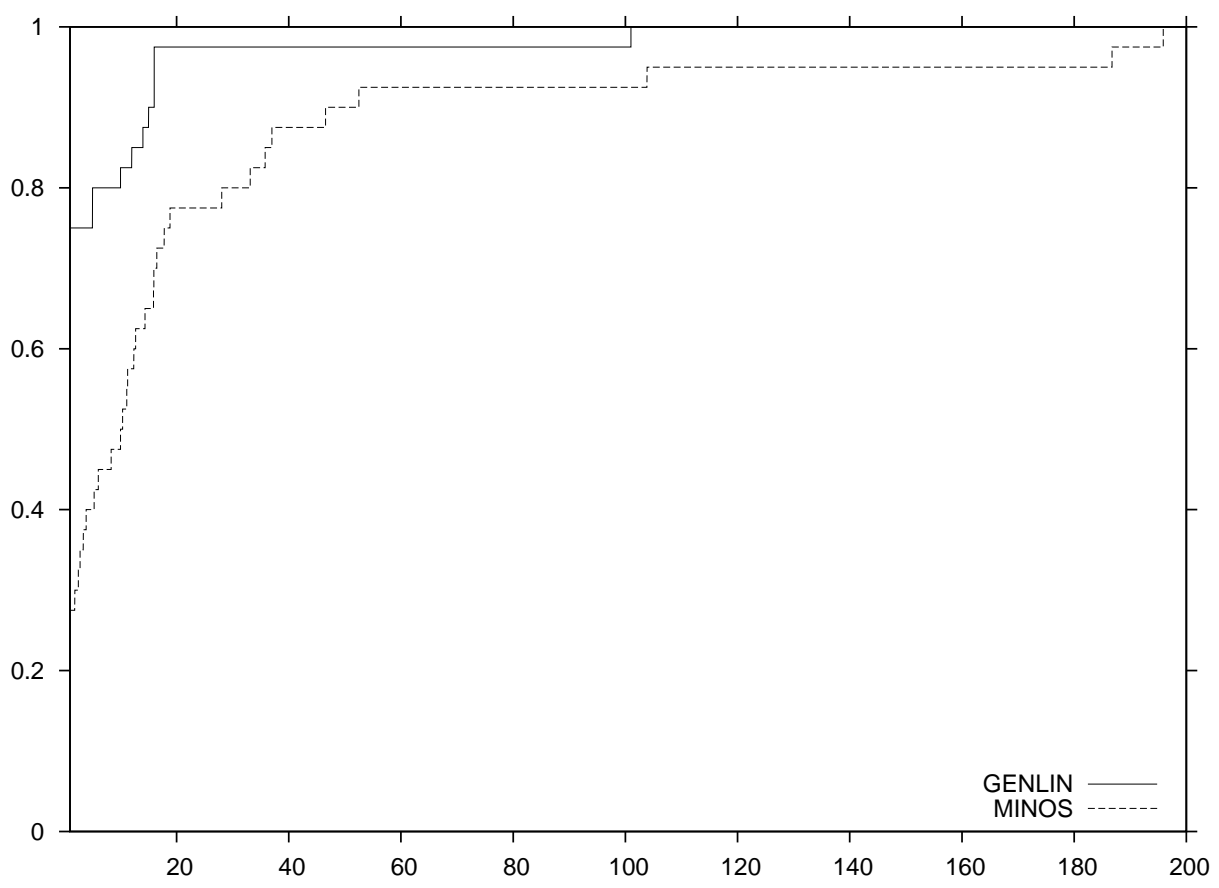

Figure 4: Performance profiles of GENLIN and Minos in the 111 problems (out of 127) for which both methods obtained feasible points with equivalent functional values. Number of functional evaluations is being used as performance measurement.

3. GEnLin and Minos performed the same number of function evaluations in one case.

4. Considering the problems in which one of the methods used at least 0.01 seconds $(40$ problems) GEnLin was faster than Minos (up to a tolerance of 10\%) 17 times and Minos was faster than GENLIN (up to a tolerance of 10\%) 22 times (both methods spent the same amount of time in one case).

5. Restricting the item above to the problems in which one of the methods used at least 0.1 seconds (27 problems) GEnlin was faster than Minos 8 times and Minos was faster than GENLIN 18 times (both methods spent the same amount of time in one case).

A performance profile using the number of functional evaluations as a performance measurement can be seen in Figure 4. We recall that, due to the use of sparsity techniques in Minos (and not in GenLin), computer time comparisons have little significance.

\subsection{Comparison with Ipopt}

IPOPT [61] is one of the best-qualified interior point methods for large-scale constrained optimization. We ran this algorithm using all its default parameters.

We applied IPOPT to the 127 feasible problems of Table 1 (details can be found in [7]). The final iterate of IPOPT in one problem (HIMMELBJ) does not satisfy the feasibility requirement with tolerance $\varepsilon_{\text {feas }}=10^{-8}$. In this case IPOPT stops declaring that restoration failed. 
In the other 126 problems, the final iterate of IPOPT is feasible with tolerance $\varepsilon_{\text {feas }}=10^{-8}$. In 121 out of those 126 problems, IPOPT stops satisfying its stopping criterion related to "success". For the remaining 5 problems, the diagnostics were:

- In problems EQC and DALLASM, IPOPT stops declaring that the problem was solved to an acceptable level.

- In problem STATIC3, IPOPT stops declaring that the problem might be unbounded.

- In problem QCNEW, IPOPT stops declaring that restoration failed.

- In problem LIN, IPOPT stops declaring that search direction is becoming too small.

Considering the 126 problems in which IPOPT obtained feasible final points, we observed that, in most of them, the final functional value at the final point is equivalent to the one obtained by GenLIN. The exceptions were:

1. In 12 problems (hS44, EXPFita, EXPFitb, EXPFITC, HS268, s268, HS55, PENTAGON, EQC, MAKELA4, GOFFIN, PRIMAL1) GENLIN obtained a smaller final functional value than IPOPT.

2. In 17 problems (BIGGSC4, HATFLDH, HS54, QCNEW, HS105, DUALC1, DEGENLPA, DEGENLPB, QPCBLEND, QPNBLEND, QPNBOEI2, GMNCASE1, GMNCASE2, GMNCASE3, PRIMALC5, QPNBOEI1, STEENBRD) IPOPT obtained a smaller final functional value than GENLIN.

3. In one problem (LIN) the final functional value provided by IPOPT was not defined (NaN message).

In the remaining 95 problems (excluding here problem sTATIC3 for which both methods detected, by different ways, that it seems to be unbounded) GENLIN and IPOPT obtained feasible points with equivalent functional values. Considering these 95 problems:

1. Genlin performed less function evaluations in 71 cases.

2. IPOPT performed less function evaluations in 15 cases.

3. GENLin and IpOpt performed the same number of function evaluations in 9 cases.

4. Considering the problems in which one of the methods used at least 0.01 seconds (54 problems) GEnLIn was faster than IPOPT (up to a tolerance of 10\%) 37 times and IPOPT was faster than GENLIN (up to a tolerance of 10\%) 15 times (both methods spent the same amount of time in 2 cases).

5. Restricting the item above to the problems in which one of the methods used at least 0.1 seconds (26 problems) GENLIN was faster than IPOPT 11 times and IPOPT was faster than GENLIN 14 times (both methods spent the same time in 2 cases).

A performance profile using the number of functional evaluations as a performance measurement can be seen in Figure 5. As in the case of Minos, we recall that time comparisons have little significance in this case since IPOPT uses sparse matrix techniques for linear algebra computations. 


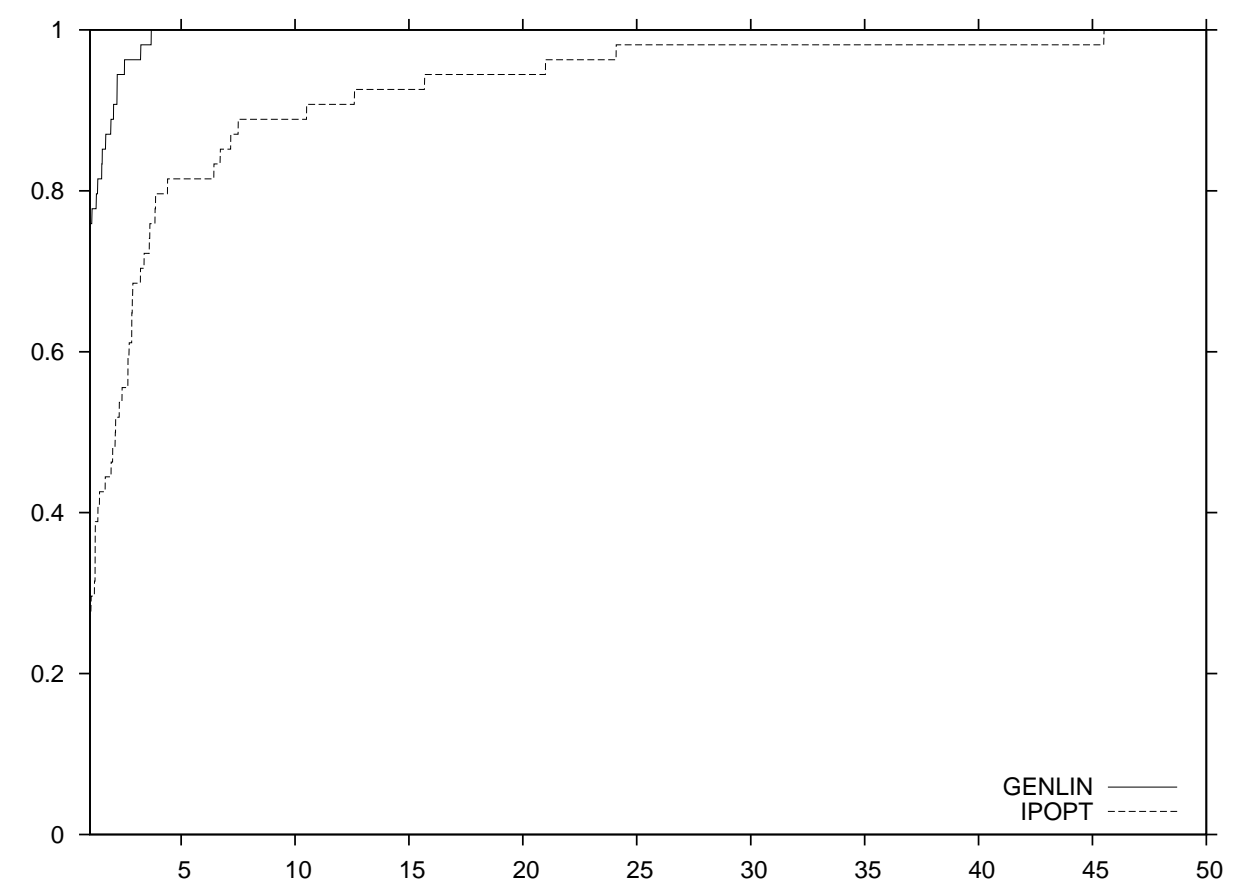

Figure 5: Performance profiles of GENLIN and IPOPT in the 95 problems (out of 127) for which both methods obtained feasible points with equivalent functional values. Number of functional evaluations is being used as performance measurement.

\subsection{Conclusions of the Numerical Experiments}

One should be very cautious when stating conclusions from very preliminar numerical experience. It must be warned that conclusions are restricted to the particular set of problems addressed and that a thorough evaluation comes only after thousands of tests made by other users. We hope that public availability of our codes may encourage some researchers (especially people involved with applications) to use them for solving their own problems. Nevertheless, the following statements seem to be well supported by the performed numerical experiments.

1. Genlin and Betralin have similar behavior both in terms of robustness and efficiency.

2. GENLIN presents a good adequacy of numerical results to theoretical convergence proofs, in the sense that, except in two cases, it stopped satisfying the predicted convergence criterion $\left\|g_{P}\left(x^{k}, \delta^{k}\right)\right\|_{\infty} \leq \varepsilon$. The exceptions were a problem in which the code stopped because the functional values seemed to tend to $-\infty$ and a problem with an undefined objective function at some trial points.

3. We think that the most interesting positive observation coming from comparative experiments is the frequency in which GENLIN obtains solutions using less function evaluations than the competitors. In the case of VE11 and Minos this can be partially attributed to the fact that these codes use quasi-Newton directions, whereas GenLIN employs truncated Newton steps. However, this not the case of IPOPT, which uses Newtonian search directions. Therefore, the efficiency in terms of functional evaluations seems to rely on the strategy for dealing with linear constraints. 


\section{$7 \quad$ Final Remarks}

As so happens to occur with unconstrained and box-constrained optimization, improvements in the techniques for minimizing smooth functions with linear constraints may cause almost immediate benefical effects in other practical optimization areas. Penalty and Augmented Lagrangian methods may solve problems with linear and nonlinear constraints placing the linear ones in a lower level and reserving the penalty-dual approach only for the nonlinear constraints $[3,21]$. Inexact-Restoration techniques [39, 46, 47] and the traditional methods of GRG type $[1,2,34]$ use to employ linearly constrained minimization subproblems for their general nonlinearly constrained calculations, and many other examples can be found in the literature involving nontraditional optimization-like problems. The potentially multiplicative aspect of this research is, therefore, one of the reasons for pursuing increasingly efficient algorithms for linearly constrained optimization. The other reason is, of course, that many Physical, Engineering and Economic problems admit linearly constrained optimization as natural mathematical models.

In this paper we made the first practical and computational attempt to incorporate the well established SPG ideas to an efficient software dedicated to optimization on polytopes without special structure. Although our final goal is to address efficiently large-scale problems, we felt the necessity of producing an acceptable piece of software for small to moderate size problems. Many algorithmic decisions were taken in the process of developing this software and some crucial features of the algorithm were introduced during the testing period. As a whole, we think that the results were pleasantly good although, as mentioned in Section 6, only thousands of tests performed by other users can confirm this initial feeling. With this purpose, we made our software available through the TANGO Project web page http://www.ime.usp.br/ egbirgin/tango/ (alternatively, follow the direct link http://www.ime.usp.br/ egbirgin/sources/genlin/) and we hope that it is clear and friendly enough to be used by people having real-life problems. Software for large-scale problems, with sparse technology, will be released soon, although we expect that new algorithmic decisions will be necessary for achieving robustness and efficiency in this case.

\section{References}

[1] J. Abadie, Modification of the GRG method, RAIRO Operations Research 3, pp. 323-326, 1979.

[2] J. Abadie and G. Guerrero, The General Reduced Gradient Method (GRG), the global Newton Method and their application to Mathematical Programming, RAIRO Operations Research 18, pp. 319-351, 1984.

[3] R. Andreani, E. G. Birgin, J. M. Martínez and M. L. Schuverdt, On Augmented Lagrangian methods with general lower-level constraints, SIAM Journal on Optimization 18, pp. 12861309, 2007.

[4] R. Andreani, E. G. Birgin, J. M. Martínez, and M. L. Schuverdt, Augmented Lagrangian methods under the Constant Positive Linear Dependence constraint qualification, Mathematical Programming 111, pp. 5-32, 2008.

[5] R. Andreani, E. G. Birgin, J. M. Martínez, and J-Y. Yuan, Spectral projected gradient and variable metric methods for optimization with linear inequalities, IMA Journal of Numerical Analysis 25 pp. 221-252, 2005. 
[6] M. Andretta, E. G. Birgin and J. M. Martínez, Practical active-set Euclidian trust-region method with spectral projected gradients for bound-constrained minimization, Optimization 54, pp. 305-325, 2005.

[7] M. Andretta, E. G. Birgin and J. M. Martínez, Partial Spectral Projected Gradient Method with Active-Set Strategy for Linearly Constrained Optimization, Technical Report MCDO090309 (see http://www.ime.usp.br/ egbirgin/), Department of Applied Mathematics, UNICAMP, Brazil, 2009.

[8] J. Barzilai and J. M. Borwein, Two point step size gradient method, IMA Journal of Numerical Analysis 8, pp. 141-148, 1988.

[9] L. Bello and M. Raydan, Convex constrained optimization for the seismic reflection tomography problem, Journal of Applied Geophysics 62, pp. 158-166, 2007.

[10] D. P. Bertsekas, On the Goldstein-Levitin-Polyak gradient projection method, IEEE Transactions on Automatic Control 21, pp. 174-184, 1976.

[11] E. G. Birgin, R. Biloti, M. Tygel, and L. T. Santos, Restricted optimization: a clue to a fast and accurate implementation of the Common Reflection Surface method, Journal of Applied Geophysics 42, pp. 143-155, 1999.

[12] E. G. Birgin, R. Castillo, and J. M. Martínez, Numerical comparison of Augmented Lagrangian algorithms for nonconvex problems, Computational Optimization and Applications 31, pp. 31-56, 2005.

[13] E. G. Birgin and Y. G. Evtushenko, Automatic differentiation and spectral projected gradient methods for optimal control problems, Optimization Methods and Software 10, pp. 125-146, 1998.

[14] E. G. Birgin and J. M. Martínez, A box-constrained optimization algorithm with negative curvature directions and spectral projected gradients, Computing [Suppl] 15, pp. 49-60, 2001.

[15] E. G. Birgin and J. M. Martínez, Large-scale active-set box-constrained optimization method with spectral projected gradients, Computational Optimization and Applications 23, pp. 101-125, 2002.

[16] E. G. Birgin and J. M. Martínez, Structured minimal-memory inexact quasi-Newton method and secant preconditioners for Augmented Lagrangian Optimization, Computational Optimization and Applications 39, pp. 1-16, 2008.

[17] E. G. Birgin, J. M. Martínez, and M. Raydan, Nonmonotone spectral projected gradient methods on convex sets, SIAM Journal on Optimization 10, pp. 1196-1211, 2000.

[18] E. G. Birgin, J. M. Martínez, and M. Raydan, Algorithm 813: SPG - Software for convexconstrained optimization, ACM Transactions on Mathematical Software 27, pp. 340-349, 2001.

[19] E. G. Birgin, J. M. Martínez, and M. Raydan, Inexact Spectral Projected Gradient methods on convex sets, IMA Journal on Numerical Analysis 23, pp. 539-559, 2003. 
[20] I. Bongartz, A. R. Conn, N. I. M. Gould, and Ph. L. Toint, CUTE: constrained and unconstrained testing environment, ACM Transactions on Mathematical Software 21, pp. 123-160, 1995.

[21] A. R. Conn, N. I. M. Gould, A. Sartenaer, and Ph. L. Toint, Convergence properties of an Augmented Lagrangian algorithm for optimization with a combination of general equality and linear constraints, SIAM Journal on Optimization 6, pp. 674-703, 1996.

[22] D. Cores and M. Loreto, A generalized two-point ellipsoidal anisotropic ray tracing for converted waves, Optimization and Engineering 8, pp. 373-396, 2007.

[23] Y. H. Dai, Alternate step gradient method, Optimization 52, pp. 395-415, 2003.

[24] Y. H. Dai and R. Fletcher, Projected Barzilai-Borwein methods for large-scale boxconstrained quadratic programming, Numerische Mathematik 100, pp. 21-47, 2005.

[25] Y. H. Dai and R. Fletcher, On the asymptotic behaviour of some new gradient methods, Mathematical Proramming 103 pp. 541-559, 2005.

[26] Y. H. Dai and R. Fletcher, New algorithms for single linearly constrained quadratic programs subject to lower and upper bounds, Mathematical Proramming 106 pp. 403-421, 2005.

[27] Y. H. Dai, W. W. Hager, K. Schittkowski, and H. C. Zhang, The cyclic Barzilai-Borwein method for unconstrained optimization, IMA Journal of Numerical Analysis 26, pp. 604$627,2006$.

[28] Y. H. Dai and H. C. Zhang, Adaptive two-point stepsize gradient algorithm, Numerical Algorithms 27, pp. 377-385, 2001.

[29] G. P. Deidda, E. Bonomi, and C. Manzi, Inversion of electrical conductivity data with Tikhonov regularization approach: some considerations, Annals of Geophysics 46, pp. 549$558,2003$.

[30] M. A. Diniz-Ehrhardt, M. A. Gomes-Ruggiero, J. M. Martínez, and S. A. Santos, Augmented Lagrangian algorithms based on the spectral projected gradient for solving nonlinear programming problems. Journal of Optimization Theory and Applications 123, pp. 497-517, 2004.

[31] E. D. Dolan and J. J. Moré, Benchmarking optimization software with performance profiles, Mathematical Programming 91, pp. 101-213, 2002.

[32] R. Fletcher, On the Barzilai-Borwein method, Department of Mathematics, University of Dundee, NA/207, Dundee, Scotland, 2001.

[33] A. Friedlander, J. M. Martínez, B. Molina, and M. Raydan, Gradient method with retards and generalizations, SIAM Journal on Numerical Analysis 36, pp. 275-289, 1998.

[34] D. Gabay and D. G. Luenberger, Efficiently converging minimization methods based on reduced gradient, SIAM Journal on Control 14, pp. 42-61, 1976. 
[35] P. E. Gill, G. H. Golub, W. Murray, and M. A. Saunders, Methods for modifying matrix factorizations, Mathematics of Computation 28, pp. 505-535, 1974.

[36] D. Goldfarb and A. Idnani, A numerically stable dual method for solving strictly convex quadratic programs, Mathematical Programming 27, pp. 1-33, 1983.

[37] A. A. Goldstein, Convex Programming in Hilbert Space, Bulletin of the American Mathematical Society 70, pp. 709-710, 1964.

[38] M. A. Gomes-Ruggiero, J. M. Martínez, and S. A. Santos, Spectral Projected Gradient Method with Inexact Restoration for Minimization with Nonconvex Constraints, SIAM Journal on Scientific Computing 31, pp. 1628-1652, 2009.

[39] C. C. Gonzaga, E. Karas, and M. Vanti, A globally convergent filter method for nonlinear programming, SIAM Journal on Optimization 14, pp. 646-669, 2003.

[40] L. Grippo, F. Lampariello, and S. Lucidi, A nonmonotone line search technique for Newton's method, SIAM Journal on Numerical Analysis 23, pp. 707-716, 1986.

[41] L. Grippo and M. Sciandrone, Nonmonotone globalization techniques for the BarzilaiBorwein gradient method, Computational Optimization and Applications 23, pp. 143-169, 2002 .

[42] L. Grippo and M. Sciandrone, Nonmonotone derivative free methods for nonlinear equations, Computational Optimization and Applications 37, pp. 297-328, 2007.

[43] Z. Jiang, Applications of conditional nonlinear optimal perturbation to the study of the stability and sensitivity of the Jovian atmosphere, Advances in Atmospheric Sciences 23, pp. $775-783,2006$.

[44] J. J. Júdice, M. Raydan, S. S. Rosa, and S. A. Santos, On the solution of the symmetric eigenvalue complementarity problem by the spectral projected gradient algorithm, Numerical Algorithms 47, pp. 391-407, 2008.

[45] E. S. Levitin and B. T. Polyak, Constrained minimization problems, USSR Computational Mathematics and Mathematical Physics 6, pp. 1-50, 1966.

[46] J. M. Martínez, Inexact-restoration method with Lagrangian tangent decrease and new merit function for nonlinear programming, Journal on Optimization Theory and Applications 111, pp. 39-58, 2001.

[47] J. M. Martínez and E. A. Pilotta, Inexact-restoration algorithm for constrained optimization, Journal on Optimization Theory and Applications 104, pp. 135-163, 2000.

[48] J. M. Martínez, E. A. Pilotta, and M. Raydan, Spectral gradient methods for linearly constrained optimization, Journal of Optimization theory and Application 125, pp. 629$651,2005$.

[49] B. A. Murtagh and M. A. Saunders, Large-scale linearly constrained optimization, Mathematical Programming 14, pp. 41-72, 1978. 
[50] M. J. D. Powell, On the quadratic programming algorithm of Goldfarb and Idnani, Mathematical Programming Study 25, pp. 46-61, 1985.

[51] M. J. D. Powell, A tolerant algorithm for linearly constrained optimization calculations, Mathematical Programming 45, pp. 547-566, 1989.

[52] A. Ramirez-Porras and W. E. Vargas-Castro, Transmission of visible light through oxidized copper films: feasibility of using a spectral projected gradient method, Applied Optics 43, pp. $1508-1514,2004$.

[53] M. Raydan, On the Barzilai and Borwein choice of steplength for the gradient method, IMA Journal of Numerical Analysis 13, pp. 321-326, 1993.

[54] M. Raydan, The Barzilai and Borwein gradient method for the large scale unconstrained minimization problem, SIAM Journal on Optimization 7, pp. 26-33, 1997.

[55] M. Raydan and B. F. Svaiter, Relaxed steepest descent and Cauchy-Barzilai-Borwein method, Computational Optimization and Applications 21, pp. 155-167, 2002.

[56] J. B. Rosen, The Gradient Projection method for Nonlinear Programming 2. Linear constraints, Journal of the Society for Industrial and Applied Mathematics 8, pp. 181-217, 1960.

[57] K. Schittkowski, QL: a Fortran code for convex quadratic programming, User's Guide, Version 2.1, 2004.

[58] T. Serafini, G. Zanghirati, and L. Zanni, Gradient projection methods for quadratic programs and applications in training support vector machines, Optimization Methods and Software 20, pp. 347-372, 2005.

[59] Ph. L. Toint, An assessment of non-monotone linesearch techniques for unconstrained optimization, SIAM Journal on Scientific Computing 17, pp. 725-739, 1996.

[60] W. E. Vargas, D. E. Azofeifa, and N. Clark, Retrieved optical properties of thin films on absorbing substrates from transmittance measurements by application of a spectral projected gradient method, Thin Solid Films 425, pp. 1-8, 2003.

[61] A. Wächter and L. T. Biegler, On the implementation of an interior-point filter line-search algorithm for large-scale nonlinear programming, Mathematical Programming 106, pp. 25$57,2006$.

[62] C. Y. Wang and Q. Liu, Convergence properties of inexact projected gradient methods, Optimization 55, pp. 301-310, 2006.

[63] C. Y. Wang, Q. Liu, and X. M. Yang, Convergence properties of nonmonotone spectral projected gradient methods, Journal of Computational and Applied Mathematics 182, pp. 51-66, 2005.

[64] Y-X. Yuan, A new stepsize for the steepest descent method, Journal of Computational Mathematics 24, pp. 149-156, 2006. 
[65] N. Zeev, O. Savasta, and D. Cores, Non-monotone spectral projected gradient method applied to full waveform inversion Geophysical Prospecting 54, pp. 525-534, 2006.

[66] H. Zhang and W. W. Hager, A nonmonotone line search technique and its application to unconstrained optimization, SIAM Journal on Optimization 14, pp. 1043-1056, 2004.

[67] L. Zhang and W. J. Zhou, Spectral gradient projection method for solving nonlinear monotone equations, Journal of Computational and Applied Mathematics 196, pp. 478-484, 2006. 